\title{
RASTREAMENTO GLOBAL VIA CONTROLE POR MODOS DESLIZANTES E OBSERVADOR COM GANHO DINÂMICO
}

\author{
Alessandro Jacoud Peixoto* \\ jacoud@poli.ufrj.br \\ Tiago Roux Oliveira
tiagoroux@uerj.br \\ Liu $\mathrm{Hsu}^{\ddagger}$ \\ liu@coep.ufrj.br \\ ${ }^{*}$ Dep. de Engenharia Eletrônica e de Computação - DEL e ${ }^{\ddagger}$ Programa de Engenharia Elétrica - PEE/COPPE \\ Universidade Federal do Rio de Janeiro - UFRJ \\ Rio de Janeiro, RJ, Brasil \\ ${ }^{\dagger}$ Dep. de Engenharia Eletrônica e Telecomunicações - DETEL \\ Universidade do Estado do Rio de Janeiro - UERJ \\ Rio de Janeiro, RJ, Brasil
}

\begin{abstract}
Global Tracking via Sliding Mode Control and Dynamic Gain Observer

A novel output-feedback sliding mode control (SMC) strategy is proposed for a class of single-input-singleoutput (SISO) uncertain time-varying nonlinear systems for which a norm state estimator can be implemented. Such a class encompasses minimum-phase systems with nonlinearities affinely norm bounded by unmeasured states with growth rate depending nonlinearly on the measured system output and on the internal states related with the zero-dynamics. The sliding surface is generated by using the state of a high gain observer (HGO) while a peaking free control amplitude is obtained via norm observer. In contrast to the existing semi-global SMC solutions available in the literature for the class of plants considered here, the proposed scheme is free of peaking and achieves global tracking with respect to a small residual set. The key idea is to design a timevarying HGO gain implementable only from measurable signals.
\end{abstract}

Artigo submetido em 29/03/2010 (Id.: 01122)

Revisado em 20/09/2010

Aceito sob recomendação do Editor Associado Prof. Luis Fernando Alves Pereira
KEYWORDS: uncertain nonlinear systems, sliding mode control, output-feedback, global tracking, high gain and norm observer.

\section{RESUMO}

Uma nova estratégia de controle por modos deslizantes via realimentação de saída é proposta para uma classe de sistemas não-lineares incertos, monovariáveis e variantes no tempo, para a qual um observador da norma do estado possa ser implementado. Esta classe inclui sistemas de fase-mínima com não-linearidades limitadas de modo afim nos estados não-medidos e com taxa de crescimento dependendo não-linearmente da saída medida e de estados internos relacionados com a dinâmica dos zeros. A superfície de deslizamento é gerada utilizando-se os estados de um observador de alto ganho (HGO), enquanto que a amplitude de controle livre de peaking é obtida a partir de um observador da norma do estado. Diferentemente das outras soluções semi-globais encontradas na literatura de controle por modos deslizantes disponíveis para a classe de plantas considerada aqui, o esquema proposto é livre de peaking e alcança o rastreamento global com respeito a um pequeno conjunto residual. A idéia chave é projetar um HGO com ganho 
variável, implementado a partir de sinais medidos.

PALAVRAS-CHAVE: sistemas não-lineares incertos, controle por modos deslizantes, realimentação de saída, rastreamento global, observador de alto ganho e da norma.

\section{INTRODUÇÃO}

Diversas abordagens para lidar com o problema de rastreamento via controle por modos deslizantes e realimentação de saída (output-feedback sliding mode (OFSM) control) para sistemas incertos com grau relativo arbitrário vêm sendo propostas na literatura de controle (Yu \& Xu, 2002; Edwards et al., 2006; Sabanovic et al., 2004; Hsu et al., 2002; Hsu et al., 2006). Dentre essas estratégias, destacam-se aquelas que utilizam HGOs (Oh \& Khalil, 1997; Cunha et al., 2009) no projeto de controle. Outras abordagens baseadas no controle por modos deslizantes de alta ordem (higher order sliding mode - HOSM) conseguem atingir rastreamento exato através dos bem conhecidos diferenciadores robustos e exatos (robust exact differentiators - RED) de Levant (2003). Contudo, propriedades de estabilidade e/ou convergência desses esquemas de controle são garantidas apenas localmente. Como discutido na introdução deste artigo, a maioria das estratégias baseadas em OFSM obtém resultados globais apenas sob severas hipóteses tais como campos vetoriais limitados linearmente ou uniformemente limitados (Hsu et al., 2002; Hsu et al., 2006; Cunha et al., 2009).

Plantas não-lineares mais gerais foram tratadas em (Hsu et al., 2006; Oh \& Khalil, 1997; Esfandiari \& Khalil, 1992; Oliveira et al., 2007; Oliveira et al., 2010a), entretanto apenas rastreamento semi-global pôde ser concluído. Isto não é surpreendente visto que, como mostrado em (Mazenc et al., 1994), para sistemas com não-linearidades polinomiais nos estados não-medidos, o problema de estabilização ou rastreamento global via realimentação de saída pode não ter solução. Além do controle por modos deslizantes, muitas outras abordagens para solucionar este problema foram propostos baseados em backstepping, HGOs com ganho variável (Praly, 2001; Krishnamurthy et al., 2002; Krishnamurthy et al., 2003; Lei \& Lin, 2005; Ahrens \& Khalil, 2007), homogeneidade (Andrieu et al., 2007; Andrieu et al., 2009) ou algum tipo de adaptação (Marino \& Tomei, 1995). Contudo, nenhum resultado global foi apresentado para a classe de sistemas considerada no presente artigo no domínio de OFSM, onde a robustez e a possibilidade de obter excelentes respostas transitórias são vantagens reconhecidas e importantes.

Acredita-se que a classe de sistemas abordada aqui está no estado da arte do controle por realimentação de saída com resultados globais comumente considerada por outros autores (Praly, 2001; Lei \& Lin, 2005; Andrieu et al., 2007; Andrieu et al., 2009; Praly \& Jiang, 2004; Kaliora et al., 2006). Nós tratamos de plantas não-lineares variantes no tempo, de fase mínima, afins no controle, transformáveis para a forma normal e para as quais é possível implementar um observador da norma do estado. Essa classe inclui sistemas nas formas outputfeedback e parametric strict feedback, sistemas triangulares com condição de crescimento linear no estado nãomedido e taxa de crescimento possivelmente dependente de estados da dinâmica interna, da saída e do tempo. Não-linearidades polinomiais nos estados não-medidos da dinâmica interna e da saída da planta são também permitidas.

Neste artigo, nós estendemos a aplicabilidade dos resultados utilizando HGO com ganho dinâmico originalmente propostos por Peixoto et al. (2007), e anteriormente restritos a sistemas com crescimento linear nos estados não-medidos e taxa de crescimento constante e conhecida, para uma classe mais ampla de nãolinearidades. O resultado principal é mostrar que o controle por OFSM baseado em um HGO com ganho dinâmico pode também ser usado para a classe no estado da arte de sistemas não-lineares, garantindo rastreamento global e prático. Para obter este novo resultado, a principal modificação com relação ao esquema proposto em Peixoto et al. (2007) reside na capacidade de se obter um majorante para a norma do estado da classe de sistemas não-lineares mais geral considerada neste trabalho, por meio de uma nova formulação de um estimador para a norma do estado. Isto permite desenvolver uma novo projeto para o ganho variante no tempo do HGO. Note que, diferentemente da maioria dos esquemas existentes, o ganho do HGO não é atualizado através da solução da equação de Riccati (Praly, 2001; Andrieu et al., 2007; Kaliora et al., 2006) mas, ao contrário, nós utilizamos funções simples (e.g., polinomiais) baseadas em sinais medidos, além de técnicas de majoraçãodominação (domination) (Lei \& Lin, 2005; Andrieu et al., 2007; Andrieu et al., 2009). Aparentemente, esse é o primeiro trabalho a conseguir esses resultados em OFSM para a classe de plantas aqui considerada.

Uma desvantagem das estratégias de controle baseadas em HGO é o fenômeno de peaking (Sussmann \& Kokotović, 1991), que pode degradar o desempenho do sistema ou mesmo levar à instabilidade. Técnicas para se evitar o peaking através de saturação do sinal de controle já haviam sido propostas em (Oh \& Khalil, 1997; Esfandiari \& Khalil, 1992), porém essa abordagem leva apenas a resultados locais ou semi-globais. Aqui, seguindo 
(Cunha et al., 2009), o peaking no sinal de controle é evitado utilizando-se sinais medidos que não são baseados em alto ganho para gerar a magnitude da lei de controle por modos deslizantes. As estimativas do HGO são utilizadas apenas para formar a superfície de deslizamento. Desta forma, um eventual peaking (Sussmann \& Kokotović, 1991) na variável de deslizamento é bloqueado pela convencional função sinal utilizada para implementar o sinal de controle por modos deslizantes, visto que a magnitude do sinal de controle é função apenas de sinais disponíveis bem condicionados (sem peaking).

Estabilidade global com respeito a um conjunto compacto e convergência exponencial para um conjunto residual pequeno no espaço de estado do erro são provadas. Exemplos acadêmicos ilustram a classe de sistemas e o comportamento dinâmico do ganho do HGO.

\section{PRELIMINARES}

As seguintes notações e terminologia serão utilizadas ao longo do texto:

- A norma-2 (Euclidiana) de um vetor $x=$ $\left[\begin{array}{llll}x_{1} & x_{2} & \cdots & x_{n}\end{array}\right]^{T}$ e a correspondente norma induzida de uma matriz $A$ são denotadas por $|x|$ e $|A|$, respectivamente. O símbolo $\lambda[A]$ denota o espectro de $A$ e $\lambda_{m}[A]=-\max _{i}\{\operatorname{Re}\{\lambda[A]\}\}$.

- A norma $\mathcal{L}_{\infty e}$ de um sinal $x(t) \in \mathbb{R}^{n}$ é definida como $\left\|x_{t}\right\|:=\sup _{0 \leq \tau \leq t}|x(\tau)|$.

- Funções classe $\mathcal{K}$ e $\mathcal{K}_{\infty}$ são definidas de acordo com (Khalil, 2002, p. 144). ISS, OSS e IOSS significam Input-State-Stable (ou Stability), OutputState-Stable (ou Stability) e Input-Output-StateStable, respectivamente (Sontag \& Wang, 1995).

- (i) $\alpha$ denota funções classe- $\mathcal{K}$; (ii) $\beta$ denota funções classe- $\mathcal{K}_{\infty}$; (iii) $\pi$ denota funções classe- $\mathcal{K} \mathcal{L}$; (iv) $\Psi$ denota funções classe- $\mathcal{K}$ conhecidas; (v) $\varphi$ e $\bar{\varphi}$ denotam funções não-negativas conhecidas.

Considere sistemas não-lineares SISO da forma

$$
\begin{aligned}
& \dot{x}=f(x, t)+g(x, t) u, \\
& y=h(x, t),
\end{aligned}
$$

onde $u \in \mathbb{R}$ é a entrada de controle (descontínua), $y \in \mathbb{R}$ é a saída medida, $x$ é o estado e as funções incertas $f(\cdot, \cdot), g(\cdot, \cdot)$ e $h(\cdot, \cdot)$ são suficientemente suaves para garantir existência local e unicidade de solução a partir de qualquer condição inicial $\left(x_{0}, t_{0}\right)$. Para cada solução de (1) existe um intervalo de tempo máximo de definição dado por $\left[0, t_{M}\right)$, onde $t_{M}$ pode ser finito ou infinito.
Portanto, o escape em tempo finito não pode ser excluído a priori. A definição de solução de Filippov é adotada (Filippov, 1964), assim como o conceito de controle equivalente estendido (Hsu et al., 2002, Section 2.3). Denotase o sinal de controle equivalente (contínuo por partes) simplesmente por $u(t)$.

Nossa estratégia de realimentação de saída conta com a implementação de um observador ou estimador da norma do estado da planta $x$. Na definição a seguir:

(i) seja $u$ a entrada da planta;

(ii) y é a saída da planta;

(iii) $\gamma_{o}$ é uma função suave;

(iv) $\varphi_{o}(\cdot, \cdot, t)$ e $\bar{\varphi}_{o}(\cdot, t)$ são funções não-negativas, contínuas por partes e limitadas em $t$ (como definido em (Kaliora et al., 2006)), além de serem contínuas em seus outros argumentos.

Definição 1 Um observador da norma para o sistema (1)-(2) é um sistema dinâmico de ordem- $m$ da forma:

$$
\begin{aligned}
& \tau_{1} \dot{\omega}_{1}=-\omega_{1}+u, \\
& \tau_{2} \dot{\omega}_{2}=\gamma_{o}\left(\omega_{2}\right)+\tau_{2} \varphi_{o}\left(\omega_{1}, y, t\right),
\end{aligned}
$$

com estados $\omega_{1} \in \mathbb{R}, \omega_{2} \in \mathbb{R}^{m-1}$ e constantes positivas $\tau_{1}, \tau_{2}$ tais que para $t \in\left[0, t_{M}\right)$ :

(i) se $\left|\varphi_{o}\right|$ é uniformemente limitada por uma constante $c_{o}>0$, então $\left|\omega_{2}\right|$ pode escapar no máximo exponencialmente e existe $\tau_{2}^{*}\left(c_{o}\right)$ tal que a dinâmica$\omega_{2}$ é BIBS (Bounded-Input-Bounded-State) estável w.r.t. $\varphi_{o}$ para $\tau_{2} \leq \tau_{2}^{*}$;

(ii) para cada $x(0), \omega_{1}(0), \omega_{2}(0)$, existe $\bar{\varphi}_{o}$ tal que

$$
|x(t)| \leq \bar{\varphi}_{o}(\omega(t), t)+\pi_{o}(t), \quad \omega:=\left[\omega_{1} \omega_{2}^{T} y\right]^{T},
$$

onde $\pi_{o}:=\beta_{o}\left(\left|\omega_{1}(0)\right|+\left|\omega_{2}(0)\right|+|x(0)|\right) e^{-\lambda_{o} t} \mathrm{com}$ $\beta_{o} \in \mathcal{K}_{\infty}$ e constante positiva $\lambda_{o}$.

\section{FORMULAÇÃO DO PROBLEMA}

Considera-se o problema de rastreamento global de sistemas da forma (1)-(2) transformáveis para a forma normal (Khalil, 2002):

$$
\begin{aligned}
& \dot{\eta}=f_{0}(x, t), \\
& \dot{\xi}=A_{\rho} \xi+b_{\rho} k_{p}(x, t)[u+d(x, t)], \quad y=c_{\rho} \xi,
\end{aligned}
$$

onde o estado transformado é definido como

$$
\bar{x}:=\left[\begin{array}{ll}
\eta^{T} & \xi^{T}
\end{array}\right]^{T}=T(x, t) .
$$


O subsistema- $\eta$ representa a dinâmica interna com $\eta \in$ $\mathbb{R}^{n-\rho}$ e o estado da dinâmica externa $(\xi)$ é dado por

$$
\xi:=\left[\begin{array}{llll}
y & \dot{y} & \ldots & y^{(\rho-1)}
\end{array}\right]^{T} .
$$

O par $\left(A_{\rho}, b_{\rho}\right)$ está na forma canônica de Brunovsky, i.e,

$$
A_{\rho}=\left[\begin{array}{cccc}
0 & 1 & \cdots & 0 \\
\vdots & \vdots & \vdots & \vdots \\
0 & 0 & \cdots & 1 \\
0 & 0 & \cdots & 0
\end{array}\right] ; \quad b_{\rho}=\left[\begin{array}{c}
0 \\
\vdots \\
0 \\
1
\end{array}\right]
$$

e $c_{\rho}=\left[\begin{array}{llll}1 & 0 & \cdots\end{array}\right]$, além disso, $d(x, t)$ é considerada como uma perturbação não-linear casada e $k_{p}(x, t)$ é o ganho de alta frequência (high frequency gain - HFG) da planta assumido não-nulo. Note que é assumido, portanto, que a planta (1)-(2) tem um grau relativo $\rho$ forte $^{1}$.

$\mathrm{Na}$ seguinte hipótese, formula-se as restrições impostas sobre $T(x, t), k_{p}(x, t)$ e $d(x, t)$, onde a dependência em $y=h(x, t)$ é explicitamente dada a fim de obtermos majorantes menos conservadores. Antes de tudo, para $i=1,2,3$, sejam:

(a) $\varphi_{i}(|x|, y, t)$ funções não-negativas, crescentes e contínuas em $|x|$, contínuas em $y$, limitadas (majoradas) e contínuas por partes em $t$;

(b) $\bar{\varphi}_{i}(y, t)$ funções não-negativas contínuas em $y$ e limitadas e contínuas por partes em $t$;

(c) $\alpha_{i}(|x|)$ funções classe- $\mathcal{K}$ localmente Lipschitz.

Hipótese 1 Existem funções conhecidas $\varphi_{i}, \bar{\varphi}_{i}, \alpha_{i}$ e uma constante positiva conhecida $c_{p}$ tais que as seguintes desigualdades são verificadas $\forall x, y, \forall t \in\left[0, t_{M}\right)$ :

$$
\begin{aligned}
\beta_{T}(|x|)+\gamma_{T}(y, t) \leq|T(x, t)| & \leq \varphi_{1}(|x|, y, t), \\
0<c_{p} \leq k_{p}(x, t) & \leq \varphi_{2}(|x|, y, t), \\
|d(x, t)| & \leq \varphi_{3}(|x|, y, t),
\end{aligned}
$$

onde $\varphi_{i}$ satisfaz $\varphi_{i}(|x|, y, t) \leq \alpha_{i}(|x|)+\bar{\varphi}_{i}(y, t), \beta_{T}$ é alguma função classe- $\mathcal{K}_{\infty}$ e $\gamma_{T}$ é uma função escalar não-negativa e contínua em $y$ e contínua por partes e uniformemente limitada em $t$.

O limitante inferior para $|T|$ assegura que se $\bar{x}$ estiver limitado, então $x$ também estará e o limitante inferior para $k_{p}$ garante que ele é positivo (sem perda de generalidade $)^{2}$. Por outro lado, as funções que limitam

\footnotetext{
${ }^{1}$ Esta terminologia é utilizada em (Diao \& Passino, 2001), onde a dependência do tempo é considerada na então chamada "derivada de Lie modificada".

${ }^{2} \mathrm{O}$ caso com $\operatorname{sgn}\left(k_{p}\right)$ desconhecido (direção de controle desconhecida) poderia ser considerado utilizando-se a generalização da técnica de funções de monitoração para grau relativo arbitrário introduzida em (Oliveira et al., 2007).
}

superiormente ou majoram $T, k_{p}$ e $d$ são utilizadas para obtermos limitantes em norma implementáveis para $\xi, k_{p}$ e $d$ a partir do vetor de estado $\omega$ do observador da norma (3)-(4). Vale ressaltar que a transformação $T(x, t)$ não é utilizada para obter $\omega$. De modo geral, os majorantes dados na Hipótese 1 impõem restrições significativas apenas com respeito a dependência no tempo, visto que $f(x, t), g(x, t)$ e $h(x, t)$ são suficientemente suaves (por hipótese) para que $T, k_{p}$ e $d$ sejam contínuos em $x$.

Observação 1 (Forma Normal) Para plantas invariantes no tempo, a hipótese de grau relativo uniforme (Khalil, 2002; Isidori, 1995) é uma condição necessária e suficiente para a existência de uma mudança de coordenadas local (difeomorfismo local) que transforma (1)-(2) em (6)-(7). Aqui, não é requerido que o mapeamento $T(x, t)(8)$ seja invertível, mas somente uma transformação global. Uma condição suficiente para assegurar que a planta variante no tempo (1)-(2) seja transformável na forma normal é dada no Apêndice A.

Adicionalmente, assume-se que:

Hipótese 2 (Fase Mínima) Existe uma função de armazenamento $V(\eta)$ satisfazendo $\underline{\beta}(|\eta|) \leq V(\eta) \leq \bar{\beta}(|\eta|)$ $\operatorname{com} \underline{\beta}, \bar{\beta} \in \mathcal{K}_{\infty}$, tal que:

$$
\frac{\partial V}{\partial \eta} f_{0}(x, t) \leq-\beta_{0}(|\eta|)+\varphi_{0}(|\xi|, t)
$$

$\forall x, y, \forall t \in\left[0, t_{M}\right)$, para algum $\beta_{0} \in \mathcal{K}_{\infty}$ e alguma função escalar não-negativa $\varphi_{0}(|\xi|, t)$, contínua em $|\xi|$ e contínua por partes e limitada em $t$.

A Hipótese 2 garante que a dinâmica interna (6) tem uma propriedade semelhante à ISS com respeito a uma função apropriada de $\xi$ e $t$. Por essa razão, isso corresponde à uma generalização do conceito de plantas de fase mínima e possibilita concluirmos que se $\xi$ estiver uniformemente limitado, então $\eta$ também estará.

Hipótese 3 (Observabilidade da Norma) A planta (1)-(2) admite um observador da norma (Definição 1) para funções $\gamma_{o}, \varphi_{o}, \bar{\varphi}_{o}$ conhecidas e constantes $\tau_{1}, \tau_{2}>0$.

É bem conhecido da literatura que, no caso invariante no tempo, se a planta (1)-(2) for IOSS (Sontag \& Wang, 1997) então ela admite um observador da norma de acordo com a Hipótese 3. Na Seção 8, apresenta-se uma classe mais geral de plantas não-lineares e variantes no tempo para a qual é possível encontrarmos um observador da norma como (3)-(4). Tal classe engloba plantas com condição de crescimento linear nos estados nãomedidos e taxa de crescimento possivelmente dependente 
de $\eta, y$ e $t$. É importante salientar que não-linearidades fortes (polinomiais) em $\eta$ e $y$ são permitidas.

\subsection{Problema de Rastreamento Global}

O objetivo é encontrar uma lei de controle dinâmica $u$ via realimentação de saída para levar o erro de rastreamento ou de saída

$$
e(t)=y(t)-y_{m}(t)
$$

exponencialmente para zero ou alguma vizinhança pequena de zero (rastreamento prático), partindo-se de quaisquer condições iniciais da planta e do controlador e mantendo todos os sinais da malha-fechada limitados uniformemente, apesar das incertezas do sistema. A trajetória desejada $y_{m}(t)$ é assumida ser gerada pelo seguinte modelo de referência:

$\dot{\xi}_{m}=A_{m} \xi_{m}+b_{\rho} k_{m} r, \quad A_{m}=A_{\rho}+b_{\rho} K_{m}, \quad y_{m}=c_{m}^{T} \xi_{m}$,

onde $\xi_{m}:=\left[\begin{array}{llll}y_{m} & \dot{y}_{m} & \ldots & y_{m}^{(\rho-1)}\end{array}\right]^{T}, k_{m}>0$ é constante, $K_{m} \in \mathbb{R}^{1 \times \rho}$ é tal que $A_{m}$ seja Hurwitz e $r(t)$ é assumida contínua por partes e uniformemente limitada.

\subsection{Do Rastreamento para a Regulação}

Subtraindo (12) de (7) tem-se

$$
\dot{\xi}_{e}=A_{m} \xi_{e}+b_{\rho} k_{p}\left[u+d_{e}\right], \quad e=c_{m}^{T} \xi_{e},
$$

onde $\xi_{e}:=\xi-\xi_{m}$ é o estado do erro de rastreamento, $c_{m}^{T}=\left[\begin{array}{llll}1 & 0 & \cdots & 0\end{array}\right]$ (então $e=\xi_{1}-\xi_{m 1}=y-y_{m}$ ) e a perturbação equivalente de entrada $d_{e}$ é definida por

$$
k_{p} d_{e}(x, \xi, t):=k_{p} d(x, t)-K_{m} \xi-k_{m} r .
$$

O problema de rastreamento pode ser formulado como um problema de regulação que consiste em encontrar uma lei de controle por modos deslizantes via realimentação de saída (OFSM) $u$ tal que $e$ seja regulado globalmente para uma vizinhança de zero (rastreamento prático). Note que, projetar $u$ apenas para assegurar a convergência de $e$ para uma vizinhança de zero não garante que os sinais em malha fechada estejam uniformemente limitados. O problema de rastreamento prático é projetar $u$ (OFSM) de tal forma que, para quaisquer condições $x(0), \omega_{1}(0), \omega_{2}(0)$ : (i) as soluções de $(3),(4)$, (6) e (7) sejam uniformemente limitadas e (ii) a saída $e=\xi_{1}-\xi_{m 1}$ de (13), i.e., o erro de rastreamento (11), tenda para uma vizinhança de zero quando $t \rightarrow \infty$.

\subsection{Limitantes Auxiliares via Observador da Norma}

Os seguintes majorantes para $\xi, k_{p}$ e $d$ são obtidos, a menos de termos exponencialmente decrescentes, a par- tir das funções limitantes dadas na Hipótese 1 e do observador da norma formulado na Definição 1:

$$
\begin{aligned}
|\xi| & \leq \psi_{1}(\omega, t)+\pi_{1}, \\
k_{p}(x, t) & \leq \psi_{2}(\omega, t)+\pi_{1}, \\
|d(x, t)| & \leq \psi_{3}(\omega, t)+\pi_{1},
\end{aligned}
$$

onde $\psi_{i}(\omega, t):=\varphi_{i}\left(2 \bar{\varphi}_{o}, y, t\right)+\bar{\varphi}_{i}(y, t)(i=1,2,3) \mathrm{e}$ $\pi_{1}=\beta_{1}(|\omega(0)|+|x(0)|) e^{-\lambda_{o} t}$ com algum $\beta_{1} \in \mathcal{K}_{\infty}$ e $\lambda_{o}$ na Definição 1 (detalhes no Apêndice C). Assim, com $c_{p}$ definido na Hipótese 1 e a partir de (14) pode-se verificar que $\left|d_{e}\right| \leq|d|+\left(\left|K_{m}\right||\xi|+k_{m}|r|\right) / c_{p}$. Além disso, de (15) e (17), a seguinte desigualdade se verifica:

$$
\left|d_{e}(x, \xi, t)\right|+\delta \leq \varrho(\omega, t)+\pi_{2},
$$

onde $\delta$ é uma constante não-negativa arbitrária,

$$
\varrho(\omega, t):=\psi_{3}+\left(\left|K_{m}\right| \psi_{1}+k_{m}|r|\right) / c_{p}+\delta,
$$

e $\pi_{2}:=\left|K_{m}\right| \pi_{1} / c_{p}+\pi_{1}$.

\section{LEI DE CONTROLE POR MODOS DES- LIZANTES E REALIMENTAÇÃO DE SAÍDA}

Quando apenas y está disponível para realimentação, pode-se escolher

$$
\hat{\sigma}:=S \hat{\xi}_{e}=0, \quad \hat{\xi}_{e}:=\hat{\xi}-\xi_{m}
$$

como a superfície de deslizamento, onde $S$ é tal que $\left(A_{m}, b_{\rho}, S\right)$ é estritamente real positivo (strictly positive real - SPR) e $\hat{\xi}$ é uma estimativa de $\xi$ (9) fornecida pelo HGO. A lei de controle $u$ é dada por

$$
u=-\varrho(\omega, t) \operatorname{sgn}(\hat{\sigma}(t)) \text {. }
$$

Assim, definindo o erro de estimação como

$$
\tilde{\xi}_{e}:=\xi_{e}-\hat{\xi}_{e}=\xi-\hat{\xi},
$$

o seguinte lema pode ser enunciado.

\section{Lema 1 (Propriedade ISS de $\left|\tilde{\xi}_{e}\right|$ para $\xi_{e}$ )}

Considere a dinâmica que governa $\xi_{e}$ em (13) com saída $\hat{\sigma}=S \xi_{e}-S \tilde{\xi}_{e}, u$ dado em (21), $\varrho$ em (19) e $d_{e}$ em (14). Então, (13) é ISS com respeito a $\tilde{\xi}_{e}$ e a seguinte desigualdade se verifica

$$
\left|\xi_{e}(t)\right| \leq k_{e}\left|\tilde{\xi}_{e}(t)\right|+\pi_{e},
$$

sendo $\pi_{e}:=\beta_{e}\left(|\omega(0)|+|x(0)|+\left|\xi_{e}(0)\right|\right) e^{-\lambda_{e} t}, \beta_{e} \in \mathcal{K}_{\infty}$, $0<\lambda_{e}<\min \left\{\lambda_{m}\left[A_{m}\right], \lambda_{o}\right\}, \lambda_{o}$ dada na Definição $1 \mathrm{e}$ $k_{e}>0$ uma constante apropriada. 
Prova: Ver Apêndice D.

Nosso objetivo é fornecer uma estimativa $\hat{\xi}$ por meio de um HGO tal que a norma do erro de observação $\left|\tilde{\xi}_{e}(t)\right|$ seja arbitrariamente pequena e utilizar o Lema 1 para concluir o rastreamento prático e global. Como demonstrado em (Peixoto, 2007), um HGO com ganho constante não é capaz de atender de forma global o objetivo traçado para uma classe geral de plantas nãolineares e desta forma, seguindo a filosofia apresentada em (Peixoto et al., 2007), nós iremos adotar um HGO com ganho variável para atingirmos o rastreamento global.

Um eventual peaking (Sussmann \& Kokotović, 1991) em $\hat{\sigma}$ é bloqueado pela função $\operatorname{sgn}(\cdot)$ em (21) e o sinal de controle $u$ é livre de peaking visto que $\varrho(\omega, t)$ é implementado utilizando-se apenas sinais disponíveis bem condicionados (sem peaking). O esquema proposto é representado na Fig. 1.

\section{OBSERVADOR DE ALTO GANHO (HGO) COM GANHO VARIÁVEL}

O HGO é dado por

$$
\dot{\hat{\xi}}=A_{\rho} \hat{\xi}+b_{\rho} u+H_{\mu} L_{o}\left(y-c_{\rho} \hat{\xi}\right),
$$

onde $L_{o}$ e $H_{\mu}$ são dados por

$$
L_{o}:=\left[\begin{array}{lll}
l_{1} & \ldots & l_{\rho}
\end{array}\right]^{T} \text { e } H_{\mu}:=\operatorname{diag}\left(\mu^{-1}, \ldots, \mu^{-\rho}\right) .
$$

O ganho do observador $L_{o}$ é tal que $s^{\rho}+l_{1} s^{\rho-1}+\ldots+$ $l_{\rho}$ é Hurwitz. Neste artigo, ao invés de usarmos um parâmetro $\mu$ constante, introduzimos um parâmetro $\mu=$ $\mu(t) \neq 0, \forall t \in\left[0, t_{M}\right)$ variável, da forma

$$
\mu(\omega, t):=\frac{\bar{\mu}}{1+\psi_{\mu}(\omega, t)},
$$

onde $\psi_{\mu}$, chamada função de dominação, é uma função não-negativa (a ser definida) contínua em seus argumentos e $\bar{\mu}>0$ é uma constante de projeto.

Para cada trajetória do sistema, $\mu$ é absolutamente contínua e $\mu \leq \bar{\mu}$. Note que $\mu$ é limitada para $t$ em qualquer sub-intervalo finito de $\left[0, t_{M}\right)$. Portanto,

$$
\mu(\omega, t) \in[\underline{\mu}, \bar{\mu}], \quad \forall t \in\left[t_{*}, t_{M}\right),
$$

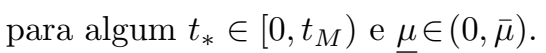

Conside a planta SISO não-linear (1)-(2) transformável na forma normal (6)-(7) sob as Hipóteses 1-3, lei de controle (21), com $\varrho$ dado por (19) e observador de alto ganho (23) com $\mu$ sendo dado por (25) e função de dominação apropriada $\psi_{\mu}$. Então, para constantes $\tau_{2}, \bar{\mu}>0$ suficientemente pequenas, são garantidas estabilidade assintótica global (global asymptotic stability GAS) do sistema do erro com respeito a um conjunto compacto e convergência exponencial do estado do sistema do erro para um conjunto residual de ordem $\bar{\mu}$, com ambos os conjuntos sendo independentes das condições iniciais do sistema. Além disso, todos os sinais do sistema em malha fechada são uniformemente limitados. A análise de estabilidade detalhada e o enunciado formal do resultado principal (Teorema 1) serão apresentados posteriormente na Seção 6 .

\subsection{Dinâmica do Erro de Observação}

A transformação (Cunha et al., 2009; Oh \& Khalil, 1997)

$$
\zeta:=T_{\mu} \tilde{\xi}_{e}, \quad T_{\mu}:=\left[\mu^{\rho} H_{\mu}\right]^{-1},
$$

é fundamental para representar a dinâmica de $\tilde{\xi}_{e}$ em um sistema de coordenadas conveniente que nos permite mostrar que $\tilde{\xi}_{e}$ fica arbitrariamente pequeno, a menos de termos exponencialmente decrescentes. Primeiro, a partir de (10), (24) e (27), note que:

$$
\begin{aligned}
T_{\mu}\left(A_{\rho}-H_{\mu} L_{o} c_{\rho}\right) T_{\mu}^{-1} & =\frac{1}{\mu} A_{o}, \\
T_{\mu} b_{\rho} & =b_{\rho}, \\
\dot{T}_{\mu} T_{\mu}^{-1} & =\frac{\dot{\mu}}{\mu} \Delta,
\end{aligned}
$$

onde

$$
A_{o}:=A_{\rho}-L_{o} c_{\rho} \quad \text { e } \quad \Delta:=\operatorname{diag}(1-\rho, 2-\rho, \ldots, 0) .
$$

Em seguida, subtraindo-se (23) de (7) e aplicando-se as relações acima, a dinâmica de $\tilde{\xi}_{e}(22)$ na nova coordenada $\zeta(27)$ é dada por:

$$
\mu \dot{\zeta}=\left[A_{o}+\dot{\mu}(t) \Delta\right] \zeta+b_{\rho}[\mu \nu]
$$

onde

$$
\nu:=\left(k_{p}-1\right) u+k_{p} d .
$$

\subsection{Projeto da Função de Dominação}

O ganho do HGO é inversamente proporcional ao pequeno parâmetro $\mu$, e é permitido ser variante no tempo a fim de garantir o rastreamento global. Nesta seção, nossa tarefa é estabelecer as propriedades que a função de dominação $\psi_{\mu}(\omega, t)$ em (25) deve possuir para que $\mu|\nu|$ e $|\dot{\mu}|$ sejam suficientemente pequenas, ao menos após um um intervalo de tempo finito. Consequentemente, após um tempo finito, $\dot{\mu}$ não afeta a estabilidade de $A_{o}$ em (31) e $\zeta$ ou $\tilde{\xi}_{e}$ podem ser feitos arbitrariamente pequenos, a menos de termos exponencialmente decrescentes. 




Figura 1: Controlador OFSM global usando HGO com ganho variável.

\subsubsection{Limitantes Superiores Auxiliares}

Note que, a partir de $(21)$, tem-se $|u(t)| \leq \varrho(\omega, t)$. Assim, a partir dos limitantes superiores (16) e (17), o sinal $\nu(32)$ satisfaz

$$
|\nu| \leq \psi_{\nu}(\omega, t)+\pi_{3},
$$

onde $\psi_{\nu}:=\varrho \psi_{2}+\varrho+\varrho^{2}+\psi_{2} \psi_{3}+\psi_{2}^{2}+\psi_{3}^{2}$ é conhecido e $\pi_{3}:=3 \pi_{1}^{2}$. Então, de $(25)$ e (33), pode-se escrever

$$
\mu|\nu| \leq \frac{\psi_{\nu}}{1+\psi_{\mu}} \bar{\mu}+\mu \pi_{3} .
$$

Com o intuito de desenvolver um majorante para $|\dot{\mu}|$, nós precisaremos de um limitante superior para $|\dot{\omega}|$. A partir de (9), tem-se $|\dot{y}| \leq|\xi|$ e, de (15), pode-se verificar que $|\dot{y}| \leq \psi_{1}(\omega, t)+\pi_{1}$. Além disso, da Definição 1 e de $(21), \dot{\omega}_{1}$ e $\dot{\omega}_{2}$ satisfazem $\tau_{1}\left|\dot{\omega}_{1}\right| \leq\left|\omega_{1}\right|+\varrho(\omega, t)$ e $\tau_{2}\left|\dot{\omega}_{2}\right| \leq\left|\gamma_{o}\left(\omega_{2}\right)\right|+\tau_{2}\left|\varphi_{o}\right|$, respectivamente. Portanto, pode-se concluir que

$$
|\dot{\omega}| \leq \psi_{\omega}(\omega, t)+\pi_{1},
$$

onde $\psi_{\omega}(\omega, t):=\psi_{1}+\left|\omega_{1}\right| / \tau_{1}+\varrho / \tau_{1}+\left|\gamma_{o}\right| / \tau_{2}+\left|\varphi_{o}\right|$ é conhecido. Finalmente, multiplicando-se (25) e (35), conseguimos

$$
\mu|\dot{\omega}| \leq \frac{\psi_{\omega}}{1+\psi_{\mu}} \bar{\mu}+\mu \pi_{1}
$$

\subsubsection{Propriedades da Função de Dominação}

Nós começamos escolhendo a função de dominação $\psi_{\mu}$ em (25) para que as seguintes propriedades sejam satisfeitas com $\psi_{\nu}$ em (33) e $\psi_{\omega}$ em (35):

(P0) $\psi_{\nu} \leq c_{\mu 0}\left(1+\psi_{\mu}\right)$ and $\psi_{\omega} \leq c_{\mu 0}\left(1+\psi_{\mu}\right), \forall t \in$ $\left[0, t_{M}\right)$, sendo $c_{\mu 0} \geq 0$ uma constante conhecida.
Se $\psi_{\mu}$ satisfaz (P0) então, a partir de 34) e (36), $\mu|\nu|$ e $\mu|\dot{\omega}|$ podem ser limitadas por

$$
\begin{aligned}
\mu|\nu| & \leq \mathcal{O}(\bar{\mu})+\mu \pi_{3} . \\
\mu|\dot{\omega}| & \leq \mathcal{O}(\bar{\mu})+\mu \pi_{1} .
\end{aligned}
$$

A fim de obtermos um majorante para $|\dot{\mu}|, \dot{\mu}$ pode ser calculado diferenciando-se (25):

$$
\dot{\mu}(t)=-\frac{\mu^{2}}{\bar{\mu}}\left[\frac{\partial \psi_{\mu}}{\partial \omega} \dot{\omega}+\frac{\partial \psi_{\mu}}{\partial t}\right]=-\frac{\frac{\partial \psi_{\mu}}{\partial \omega}}{1+\psi_{\mu}} \mu \dot{\omega}-\frac{\frac{\partial \psi_{\mu}}{\partial t}}{1+\psi_{\mu}} \mu .
$$

Note que, $\dot{\mu}$ é um sinal contínuo por partes que pode ser limitado superiormente por

$$
|\dot{\mu}(t)| \leq \frac{\left|\frac{\partial \psi_{\mu}}{\partial \omega}\right|}{1+\psi_{\mu}} \mu|\dot{\omega}|+\frac{\left|\frac{\partial \psi_{\mu}}{\partial t}\right|}{1+\psi_{\mu}} \mu .
$$

Nossa estratégia é projetar $\psi_{\mu}(\omega, t)$ tal que a seguinte propriedade adicional seja satisfeita:

(P1) $\left|\frac{\partial \psi_{\mu}}{\partial \omega}\right| \leq c_{\mu 1}\left(1+\psi_{\mu}\right)$ and $\left|\frac{\partial \psi_{\mu}}{\partial t}\right| \leq c_{\mu 1}\left(1+\psi_{\mu}\right), \forall t \in$ $\left[0, t_{M}\right)$, sendo $c_{\mu 1} \geq 0$ uma constante conhecida.

Essa propriedade é trivialmente satisfeita por uma função polinomial $\psi_{\mu}$ com coeficientes positivos (vide Seção 5.2.3).

Agora, com $\psi_{\mu}$ satisfazendo (P1), tem-se que:

$$
|\dot{\mu}(t)| \leq c_{\mu 1} \mu|\dot{\omega}|+c_{\mu 1} \mu .
$$

Consequentemente, de (41), (37) e (38) a seguinte desigualdade pode ser obtida:

$$
|\dot{\mu}(t)|, \mu|\nu| \leq \mathcal{O}(\bar{\mu})+\mu \pi_{4},
$$

onde $\pi_{4}:=c_{\mu 1} \pi_{1}+\pi_{3}$. 
Note que, a partir de (5) e da Hipótese 1, se qualquer sinal do sistema em malha fechada escapa em tempo finito, então $\omega$ também escapa. Realmente, de acordo com a Hipótese 3, o sistema goza da propriedade denominada unboundedness observability (UO) (Angeli \& Sontag, 1999). Nós usaremos este fato para projetar $\psi_{\mu}(\omega, t)$ de modo que se $\omega$ escapa em algum tempo finito então $\psi_{\mu}(\omega, t)$ também escapa não posteriormente a este instante. A partir de (25), isso irá garantir que o segundo termo no lado direito de $(42)$ será de ordem $\mathcal{O}(\bar{\mu})$, antes de qualquer eventual escape em tempo finito.

Com essa finalidade, $\psi_{\mu}$ deve ser projetado para satisfazer a propriedade:

(P2) $\left\|\omega_{t}\right\| e^{-\lambda_{\mu} t} \leq \psi_{\mu}(\omega, t), \forall \omega, \forall t \in\left[0, t_{M}\right)$, sendo $\lambda_{\mu}$ uma constante positiva de projeto.

O termo exponencial com taxa $\lambda_{\mu}$ atua como um fator de esquecimento que permite um projeto para $\psi_{\mu}$ menos conservador. Relembrando que $\pi_{4}$ pode ser escrito como $\pi_{4}=\beta_{4}(|\omega(0)|+|x(0)|) e^{-\lambda_{4} t}, \operatorname{com} \beta_{4} \in \mathcal{K}_{\infty}$ e $\lambda_{4}$ sendo uma constante positiva, então, se $\psi_{\mu}$ satisfaz (P2), a seguinte relação pode ser obtida

$$
\mu \pi_{4} \leq \bar{\mu} \frac{\pi_{4}}{1+\psi_{\mu}} \leq \bar{\mu} \frac{\beta_{4}(|\omega(0)|+|x(0)|) e^{-\lambda_{4} t}}{1+\left\|\omega_{t}\right\| e^{-\lambda_{\mu} t}},
$$

$\forall t \in\left[0, t_{M}\right)$. Pode-se mostrar que (vide Section C) o lado direito de (43) é majorado por $\bar{\mu}$, ao menos após algum tempo finito $\left(t_{\mu} \geq 0\right)$. Finalmente, se $\psi_{\mu}$ é projetado para que (P0)-(P2) sejam satisfeitas, então de (42) e (43) pode-se verificar que existe um tempo finito $t_{\mu} \in\left[0, t_{M}\right)$ tal que:

$$
\begin{gathered}
|\omega|,|\zeta| \leq \beta_{5}(|\omega(0)|+|x(0)|+|\zeta(0)|), \quad \forall t \in\left[0, t_{\mu}\right), \\
|\dot{\mu}(t)|, \mu|\nu| \leq \mathcal{O}(\bar{\mu}), \quad \forall t \in\left[t_{\mu}, t_{M}\right),
\end{gathered}
$$

com algum $\beta_{5} \in \mathcal{K}_{\infty}$. Para compreender que (44) e (45) são satisfeitas, veja o Apêndice C.

\subsubsection{Um Projeto Específico para o Ganho Dinâmico}

A hipótese a seguir é útil para determinar ao menos uma classe específica de $\mu$ 's variantes no tempo, satisfazendo as propriedades listadas acima.

Hipótese 4 Existe um polinômio $\bar{p}_{\mu}(|\omega|)$ em $|\omega|$, com coeficientes reais positivos, tal que as funções $\varphi_{o}, \bar{\varphi}_{o}$ (Hipótese 3 ) e as funções limitantes $\varphi_{i}, \bar{\varphi}_{i}$ (Hipótese 1) satisfazem $(i=1,2,3)$ :

$$
\begin{gathered}
\left|\gamma_{o}\left(\omega_{2}\right)\right|,\left|\varphi_{o}\left(\omega_{1}, y, t\right)\right| \leq \bar{p}_{\mu}(|\omega|), \\
\varphi_{i}\left(2 \bar{\varphi}_{o}(\omega, t), y, t\right), \bar{\varphi}_{i}(y, t) \leq \bar{p}_{\mu}(|\omega|) .
\end{gathered}
$$

Agora, relembrando que $\psi_{\nu}(\omega, t)$ em $(33)$ e $\psi_{\omega}(\omega, t)$ em (35) são dadas por $\psi_{\nu}(\omega, t)=\varrho \psi_{2}+\varrho+\varrho^{2}+\psi_{2} \psi_{3}+$ $\psi_{2}^{2}+\psi_{3}^{2}$ e $\psi_{\omega}(\omega, t)=\psi_{1}+\left|\omega_{1}\right| / \tau_{1}+\varrho / \tau_{1}+\left|\gamma_{o}\right| / \tau_{2}+\left|\varphi_{o}\right|$, respectivamente, onde $\psi_{i}=\varphi_{i}\left(2 \bar{\varphi}_{o}, y, t\right)+\bar{\varphi}_{i}(y, t)(i=$ $1,2,3)$ em (15)-(17). Então, com a Hipótese 4, podese facilmente obter um polinômio $p_{\mu}(|\omega|)$ em $|\omega|$, com coeficiente reais positivos, tal que:

$$
\psi_{\nu}, \psi_{\omega} \leq p_{\mu}(|\omega|) .
$$

Aqui, nós escolhemos $\psi_{\mu}$ como:

$$
\psi_{\mu}(\omega, t):=p_{\mu}(|\omega|)+\left\|\omega_{t}\right\| e^{-\lambda_{\mu} t},
$$

onde $\lambda_{\mu}>0$ é uma constante de projeto. Não é difícil verificar que (47) satisfaz (P0) e (P2). Para compreender como (P1) também é satisfeita, veja o Apêndice C.

De acordo com a Hipótese 4, assume-se a existência de um mesmo polinômio como função de majoração para as funções $\varphi_{o}, \bar{\varphi}_{o}, \varphi_{i}$ e $\bar{\varphi}_{i}$, o que pode ser conservador no sentido de se obter valores muito conservadores de $p_{\mu}$ e, consequentemente, valores muito pequenos para $\mu(t)$. Vale ressaltar que essa hipótese não é tão restritiva visto que apenas condições de crescimento polinomial são impostas à $\varphi_{o}, \bar{\varphi}_{o}, \gamma_{o}, \varphi_{i}, \bar{\varphi}_{i}$.

\section{ANÁLISE DE ESTABILIDADE E RESULTADOS PRINCIPAIS}

A fim de considerar todas as condições iniciais de todos os sinais envolvidos no sistema do erro (13) e (31), seja:

$$
z^{T}(t):=\left[z^{0}(t), \xi_{e}^{T}(t), \zeta^{T}(t)\right], z^{0}(t):=z^{0}(0) e^{-\lambda t}
$$

na qual $z^{0}(0):=\left[\eta^{T}(0) \omega^{T}(0)\right]$ e $\lambda>0$ é uma constante genérica. A análise de estabilidade do controlador em malha fechada é conduzida no Apêndice D através dos seguintes passos:

Passo-1 Primeiramente, nós demonstramos que $|z(t)|$ é uniformemente limitado por uma função classe- $\mathcal{K}_{\infty}$ de $|z(0)|, \forall t \in\left[0, t_{\mu}\right)$.

Passo-2 Depois, para $t \in\left[t_{\mu}, t_{M}\right)$ nós provamos que a norma do erro de observação é limitada por $\left|\tilde{\xi}_{e}(t)\right| \leq$ $\beta_{z 1}(|z(0)|) e^{-\lambda_{z 1} t}+\mathcal{O}(\bar{\mu})$, onde $\lambda_{z 1}>0$ é uma constante e $\beta_{z 1} \in \mathcal{K}_{\infty}$, desde que $\bar{\mu}$ seja escolhido suficientemente pequeno e $(\mathrm{P} 0)-(\mathrm{P} 1)$ sejam satisfeitas.

Passo-3 Aplicando-se o Lema 1, pode-se também verificar que $\left|\xi_{e}\right|,|z(t)| \leq \beta_{z 2}(|z(0)|) e^{-\lambda_{z 2} t}+\mathcal{O}(\bar{\mu})$, onde $\lambda_{z 2}>0$ é uma constante e $\beta_{z 2} \in \mathcal{K}_{\infty}$. Além disso, $z(t)$ não pode escapar em tempo finito. 
Passo-4 Finalmente, verifica-se que nenhum sinal da malha fechada pode escapar em tempo finito e que são uniformemente limitados $\forall t$, contanto que $\tau_{2}$ (na Definição 1) seja escolhido suficientemente pequeno.

O seguinte teorema resume o resultado principal.

Teorema 1 Considere a planta não-linear SISO (1)-(2) transformável na forma normal (6)-(7) sob as Hipóteses $1-3$. Seja a lei de controle dada por (21), com $\varrho$ em (19) e considere o observador de alto ganho (23) com $\mu$ dado por (25) e a função de dominação $\psi_{\mu}$ projetada tal que as propriedades $(\mathrm{P} 0)-(\mathrm{P} 2)$ sejam verificadas. Então, para constantes $\tau_{2}, \bar{\mu}>0$ suficientemente pequenas, existem $\beta_{z}(\cdot) \in \mathcal{K}_{\infty}$ e constantes positivas $a, b$ tais que o estado completo do erro $z$ (48) satisfaz

$$
|z(t)| \leq\left[\beta_{z}(|z(0)|)+b\right] e^{-a t}+\mathcal{O}(\bar{\mu}),
$$

$\forall t \geq 0$ e $\forall z(0)$, i.e., GAS uniforme do sistema do erro com respeito ao conjunto compacto $\{z:|z| \leq b\}$ e convergência exponencial de $z(t)$ para um conjunto residual de ordem $\mathcal{O}(\bar{\mu})$ são garantidas, com ambos os conjuntos sendo independentes das condições iniciais. Além disso, todos os sinais do sistema em malha fechada são uniformemente limitados.

\section{Prova: Ver Apêndice D.}

O fenômeno de chattering de frequência finita (Edwards \& Spurgeon, 1998) é evitado e um modo deslizante ideal é produzido graças à uma malha de deslizamento ideal formada em torno da função relé (vide Fig. 1), de acordo com o seguinte corolário.

\section{Corolário 1 (Modo Deslizante Ideal)}

Adicionalmente as hipóteses do Teorema 1 , se $\varrho \geq$ $\left|K_{m}\right|\left|\xi_{m}\right|+\left|k_{m}\right||r|+\delta$ com $\delta>0$, então o modo deslizante $\hat{\sigma} \equiv 0$ é alcançado em tempo finito.

\section{Prova: Ver Apêndice D.}

\section{Observação 2 (Chattering no Controle)}

A importância da existência do modo deslizante ideal já foi discutida em vários trabalhos, e.g., (Utkin, 1992; Bondarev et al., 1985; Hsu, 1997). Isto se deve porque, na ausência de ruído, a frequência do chattering pode ser arbitrariamente aumentada reduzindo-se o período de amostragem na implementação digital em tempo real do esquema de controle. Em muitas aplicações, como em conversores ou acionamentos elétricos, o chattering com frequência suficientemente alta é aceitável e preserva as vantagens do controle por modos deslizantes.
Ao contrário, quando filtros diferenciadores causais lineares são utilizados para reconstruir os estados requeridos na função de chaveamento $\hat{\sigma}$, pequenos e inevitáveis atrasos são introduzidos na malha de alta frequência e isso geralmente leva ao chattering com frequência limitada, independentemente do período de amostragem, o que deteriora o desempenho do controle por modos deslizantes.

Observação 3 (Ausência de Peaking) Pode-se concluir que $\xi_{e}$ é livre de peaking notando-se que (13) é ISS com respeito a $u$ e que a função $\operatorname{sgn}(\cdot)$ em $u(21)$ bloqueia qualquer eventual peaking presente em $\hat{\xi}$ para $u$.

\section{ALGORITMO DO CONTROLADOR}

O projeto completo do controlador é resumido na Tabela 1. Os parâmetros de projeto podem ser obtidos como a seguir.

Primeiramente, nós projetamos o observador da norma para a o estado da planta $x$, de acordo com a Definição 1 , e transformamos o sistema original para a forma normal. A partir da funções limitantes $\bar{\varphi}_{o}, \varphi_{i}, \bar{\varphi}_{i}(i=1,2,3)$, dadas nas Hipóteses 1-3, obtém-se: as funções $\psi_{i}$, a função de modulação (ganho de controle) (19) $\varrho$ e as funções limitantes $\psi_{\omega}$ e $\psi_{\nu}$.

Depois, projeta-se a função de dominação $\psi_{\mu}$ satisfazendo as propriedades (P0)-(P2). A constante $\lambda_{\mu} \geq 0$ é arbitrária e $L_{o}$ é tal que $s^{\rho}+l_{1} s^{\rho-1}+\ldots+l_{\rho}$ seja Hurwitz. O HGO pode ser implementado a partir de (23). Assim, a lei de controle (21) é implementada com superfície de deslizamento (20) escolhida para que $\left(A_{m}, b_{\rho}, S\right)$ seja SPR.

A partir das funções $\varphi_{o}, \gamma_{o}$ e da constante $\tau_{1}$ dada na Hipótese 3, implementa-se o observador da norma. Finalmente, por simulação, nós iniciamos o algoritmo com valores não tão pequenos de $\bar{\mu}, \tau_{2}$ e depois diminuímos $\bar{\mu}$ até que um rastreamento aceitável seja obtido. Posteriormente, nós diminuímos $\tau_{2}$ a fim de assegurar que $\omega_{2}$ seja limitado (vide Definição 1). Note que a análise de estabilidade (Teorema 1) assegura a existência de $\bar{\mu}$ e $\tau_{2}$ suficientemente pequenos para se obter rastreamento aceitável e todos os sinais em malha fechada limitados uniformemente, independentemente das condições iniciais. Entretanto, vale ressaltar que uma determinada sintonia de $\bar{\mu}, \tau_{2}$ pode ser válida apenas para as condições iniciais utilizadas no procedimento de sintonia descrito.

O projeto da função de modulação $\varrho$ pode ser conservador no sentido de gerar amplitudes elevadas do sinal de controle, podendo eventualmente saturar o atuador 
durante um transitório em um experimento real. Além disso, valores muito pequenos de $\bar{\mu}$ e $\tau_{2}$ pode inviabilizar a implementação digital do HGO e do estimador da norma, respectivamente. Por outro lado, de acordo com o Teorema 1, o erro de rastreamento converge para um conjunto residual de ordem $\mathcal{O}(\bar{\mu})$ uma vez escolhidos valores de $\bar{\mu}$ e $\tau_{2}$ suficientemente pequenos e uma função de modulação apropriada. Portanto, fica evidente os impactos do conservadorismo sobre o rastreamento.

\section{UMA CLASSE ILUSTRATIVA DE PLANTAS NÃO-LINEARES}

Nós podemos controlar plantas (1)-(2) da forma

$$
\begin{aligned}
\dot{\eta} & =\phi_{0}(x, y, t), \\
\dot{v}_{1} & =v_{2}+\phi_{1}(x, y, t), \\
\vdots & \\
\dot{v}_{\rho-1} & =v_{\rho}+\phi_{\rho-1}(x, y, t), \\
\dot{v}_{\rho} & =k_{u} u+\phi_{\rho}(x, y, t), \\
y & =v_{1},
\end{aligned}
$$

transformáveis para a forma normal (vide Observação 1) satisfazendo a Hipótese 1 . O estado $x$ é particionado em $x^{T}:=\left[\begin{array}{ll}\eta^{T} & v^{T}\end{array}\right]$, com $v \in \mathbb{R}^{\rho}$, e $k_{u}>0$ sendo uma constante. Note que esse sistema não está nem na forma triangular nem é invariante no tempo como em (Praly \& Jiang, 2004).

Agora, nós formularemos condições suficientes para $\phi^{T}=$ $\left[\begin{array}{lll}\phi_{1} & \ldots & \phi_{\rho}\end{array}\right]$ tais que $(50)-(51)$ satisfaça a hipótese de fase mínima (Hipótese 2) e a hipótese sobre a existência de um observador da norma (Hipótese 3 ).

Assim como em (Qian \& Lin, 2002; Praly \& Jiang, 2004; Choi \& Lim, 2005), considera-se que:

(C0) (Condição de Triangularidade) Para $i=1, \ldots, \rho$ :

$$
\left|\phi_{i}\right| \leq \varphi_{r}(|\eta|, y, t)\left(\left|v_{1}\right|+\ldots+\left|v_{i}\right|\right)+\varphi_{v}(|\eta|, y, t),
$$

$\forall t \in\left[0, t_{M}\right)$, onde $\bar{\varphi}_{r}, \bar{\varphi}_{v}$ são funções conhecidas nãonegativas, contínuas em $y$ e contínuas por partes e limitadas em $t$ satisfazendo $\varphi_{r}(|\eta|, y, t) \leq \Psi_{r}(|\eta|)+$ $\bar{\varphi}_{r}(y, t)$ e $\varphi_{v}(|\eta|, y, t) \leq \Psi_{v}(|\eta|)+\bar{\varphi}_{v}(y, t)$ com funções $\Psi_{r}, \Psi_{v} \in \mathcal{K}$ localmente Lipschitz também conhecidas.

Para o subsistema $\eta$, nós admitimos que pode-se obter uma função de armazenamento $V(\eta)$ satisfazendo $\underline{\alpha}(|\eta|) \leq V(\eta) \leq \bar{\alpha}(|\eta|), \operatorname{com} \underline{\alpha}(\sigma)=\underline{\lambda} \sigma^{2}, \bar{\alpha}(\sigma)=\bar{\lambda} \sigma^{2} \mathrm{e}$ $\underline{\lambda}, \bar{\lambda}$ conhecidos de modo que a seguinte condição é verificada.
(C1) Existem funções conhecidas não-negativas $\varphi_{\eta}(y, t)$, contínuas em $y$, contínuas por partes e limitadas em $t$ e um $\alpha \in \mathcal{K}$ também conhecido tal que $\forall t \in\left[0, t_{M}\right)$ :

$$
\frac{\partial V(\eta)}{\partial \eta} \phi_{0} \leq-\alpha(|\eta|)+\varphi_{\eta}(y, t)
$$

onde a função classe- $\mathcal{K} \alpha \circ \bar{\alpha}^{-1}$ é stiffening ${ }^{3}$ no intervalo $(0, \infty)$.

Note que, (C1) implica na Hipótese 2. Além disso, (C0) e (C1) nos permitem implementar o seguinte observador da norma de $3^{\underline{a}}$ ordem para $x$ :

$$
\begin{aligned}
\tau_{1} \dot{\omega}_{1} & =-\omega_{1}+u \\
\dot{\omega}_{21} & =-c_{0} \omega_{21}+\varphi_{1}(y, t), \\
\tau_{2} \dot{\omega}_{22} & =-\left(1-e^{-\omega_{22}}\right)+\tau_{2} \varphi_{2}\left(\omega_{21}\right)+\tau_{2} \varphi_{3}(y, t),
\end{aligned}
$$

que está em concordância com a Definição 1. A dinâmica de $\omega_{21}$ fornece um majorante para $|\eta|$, enquanto que a dinâmica de $\omega_{22}$ provê um majorante para $|v|$, de modo que:

$$
|x| \leq \varphi_{4}\left(\omega_{1}, \omega_{21}, y, t, \tau_{2}\right)+c_{1} e^{c_{2}\left|\omega_{22}\right|}+\pi,
$$

sendo $c_{0}, c_{1}, c_{2}$ constantes não-negativas, $\pi \quad:=$ $\beta_{0}(|\omega(0)|+|x(0)|) e^{-\lambda_{o} t}$ e $\tau_{1}, \tau_{2}, \lambda_{o}$ constantes positivas de projeto. No Apêndice B, são dados os passos necessários para obtermos as funções $\varphi_{1}, \varphi_{2}, \varphi_{3}$ e $\varphi_{4}$ do observador da norma. A seguir, ilustra-se a classe de sistemas através de um exemplo acadêmico não-trivial.

Exemplo 1 (Classe de Sistemas) Considere a seguinte planta não-linear de $4 \underline{a}$ ordem e grau relativo $\rho=3$ :

$$
\begin{aligned}
\dot{\eta} & =-\eta^{5}-|y| \eta^{2}+y \theta(t), \\
\dot{v}_{1} & =v_{2}+\eta y^{2} \\
\dot{v}_{2} & =v_{3}+\frac{v_{3}^{2}}{4+4 v_{3}^{2}} \operatorname{sen}\left(v_{2}\right)+\eta^{2} y v_{2} \\
\dot{v}_{3} & =u+\eta^{2} y v_{2}^{2 / 3} v_{3}^{1 / 3} \\
y & =v_{1}
\end{aligned}
$$

na qual $\theta(t)$ é uma função variante no tempo uniformemente limitada. Os termos não-lineares na dinâmica de $v$ satisfazem $(\mathrm{C} 0) \operatorname{com} \varphi_{r}=\eta^{2}|y|$ e $\varphi_{v}=|\eta| y^{2}+0.25$

\footnotetext{
${ }^{3}$ Assim como em (Arcak et al., 2002), nós dizemos que $\alpha_{1}(\sigma)$ é stiffening se para todo $\lambda>0$, existe $\epsilon>0$ tal que $\sigma \geq \epsilon \Rightarrow$ $\alpha_{1}(\sigma) \geq \lambda \sigma$. Uma condição menos conservadora sobre $\alpha \circ \bar{\alpha}^{-1}$ tal qual a condição ULLB (ultimately linearly lower bounded) introduzida em (Oliveira et al., 2010b; Oliveira et al., 2009) poderia ser utilizada ao invés da restrição stiffening.
} 
Tabela 1: Algoritmo proposto para atingir rastreamento global com HGO dinâmico e e controle por modos deslizantes livre de peaking.

\begin{tabular}{|c|c|}
\hline Modelo de Referência (12) & $\dot{\xi}_{m}=A_{m} \xi_{m}+b_{\rho} k_{m} r, \quad \xi_{m}:=\left[\begin{array}{llll}y_{m} & \dot{y}_{m} & \ldots & y_{m}^{(\rho-1)}\end{array}\right]^{T}$. \\
\hline Erro de Saída (11) & $e=y-y_{m}$ \\
\hline Observador da Norma (4) & $\tau_{1} \dot{\omega}_{1}=-\omega_{1}+u$ e $\tau_{2} \dot{\omega}_{2}=\gamma_{o}\left(\omega_{2}\right)+\tau_{2} \varphi_{o}\left(\omega_{1}, y, t\right) \quad($ vide Def. 1$)$ \\
\hline Limitantes Auxiliares & $\psi_{i}(\omega, t):=\varphi_{i}\left(2 \bar{\varphi}_{o}, y, t\right)+\bar{\varphi}_{i}(y, t)(i=1,2,3), \operatorname{com} \varphi_{i}, \bar{\varphi}_{i}$ em H1. \\
\hline Função de Modulação (19) & $\varrho(\omega, t):=\psi_{3}(\omega, t)+\left|K_{m}\right| \psi_{1}(\omega, t)+k_{m}|r|+\delta$ \\
\hline Função de Dominação & $\begin{array}{c}\psi_{\nu}(\omega, t):=\varrho \psi_{2}+\varrho+\varrho^{2}+\psi_{2} \psi_{3}+\psi_{2}^{2}+\psi_{3}^{2} \\
\psi_{\omega}(\omega, t):=\psi_{1}+\left|\omega_{1}\right| / \tau_{1}+\varrho / \tau_{1}+\left|\gamma_{o}\right| / \tau_{2}+\left|\varphi_{o}\right| \\
\psi_{\mu}(\omega, t) \text { satisfazendo }(\mathrm{P} 0)(\mathrm{P} 1)(\mathrm{P} 2)\end{array}$ \\
\hline HGO (23) & $\dot{\hat{\xi}}=A_{\rho} \hat{\xi}+b_{\rho} u+H_{\mu} L_{o}\left(y-c_{\rho} \hat{\xi}\right)$ \\
\hline Parâmetro $\mu(25)$ & $\begin{array}{c}L_{o}=\left[\begin{array}{lll}l_{1} & \ldots & l_{\rho}\end{array}\right]^{T}, H_{\mu}=\operatorname{diag}\left(\mu^{-1}, \ldots, \mu^{-\rho}\right) \\
\mu(\omega, t):=\frac{\bar{\mu}}{1+\psi_{\mu}(\omega, t)}, \text { sendo } \bar{\mu} \text { uma constante de projeto. }\end{array}$ \\
\hline Superfície de Deslizamento (20) & $\hat{\sigma}:=S\left(\hat{\xi}-\xi_{m}\right)=0 \operatorname{com}\left(A_{m}, b_{\rho}, S\right) \mathrm{SPR}$ \\
\hline Lei de Controle (21) & $u=-\varrho(\omega, t) \operatorname{sgn}(\hat{\sigma}(t))$. \\
\hline
\end{tabular}

e a dinâmica interna, adaptada de (Jiang et al., 2004, Ex. 1), satisfaz (C1) com $V(\eta)=\eta^{2} / 2, \alpha=|\eta|^{6} / 4 \mathrm{e}$ $\varphi_{\eta}=y^{2}\left[1+\theta^{2}\right] / 2+0.5^{\frac{1}{3}}$. O termo cruzado $v_{2}^{2 / 3} v_{3}^{1 / 3}$ foi inspirado em (Qian \& Lin, 2002, Ex. 2.4) e (Choi \& Lim, 2005). Note que o sistema é não-triangular mas é transformável para a forma normal (6)-(7). Além disso, a partir do cálculo das derivadas temporais $\dot{y}, \ddot{y}, \dddot{y}$ podese obter $T(x, t), k_{p}(x, t)$ e $d(x, t)$ satisfazendo a Hipótese 1. A Hipótese 3 também é verificada e os passos para construirmos o observador da norma (53)-(55) são fornecidos no Apêndice B.

No próximo exemplo, nós focamos apenas em observar o comportamento variante no tempo de $\mu(t)$.

Exemplo 2 (Comportamento do Parâmetro $\mu$ ) Considere o caso simples, sem dinâmica dos zeros e grau relativo dois $(\rho=2)$, onde (50)-(51) é reduzido para:

$$
\begin{aligned}
\dot{v}_{1} & =v_{2}, \\
\dot{v}_{2} & =k_{u} u-\delta_{1} v_{2}+\delta_{2} y^{2}+\delta_{3} \operatorname{sen}\left(2 \pi \delta_{4} t\right), \\
y & =v_{1} .
\end{aligned}
$$

A planta já está na forma normal (6)-(7) $(\operatorname{com} T=$ $I)$, na qual $\xi=x, k_{p}=k_{u}$ e $k_{p} d=-\delta_{1} \xi_{2}+\delta_{2} y^{2}+$ $\delta_{3} \operatorname{sen}\left(2 \pi \delta_{4} t\right)$. A Hipótese 1 é satisfeita com: $\varphi_{1}=\gamma_{T}=$ $\bar{\varphi}_{1}=1, \alpha_{1}=\beta_{T}=0, c_{p}=1, \varphi_{2}=\bar{\varphi}_{2}=2, \alpha_{2}=0$, $\alpha_{3}=3|x|, \bar{\varphi}_{3}=3 y^{2}+2$ e $\varphi_{3}=\alpha_{3}+\bar{\varphi}_{3}$.
Os parâmetros incertos são: $1 \leq k_{u} \leq 2,1 \leq \delta_{1}, \delta_{2}<$ $3,0.5 \leq \delta_{3}<2$ e $8 \leq \delta_{4} \leq 10$. Os parâmetros reais da planta, assumidos desconhecidos, são $k_{u}=2, \delta_{1}=2$, $\delta_{2}=1, \delta_{3}=0.7$ e $\delta_{4}=10$.

Naturalmente, a Hipótese 2 (e (C1)) são inexistentes neste caso. Além disso, visto que $\delta_{1}>0$, não é difícil verificar que a Hipótese 3 é satisfeita com o observador da norma de $2^{\underline{a}}$ ordem da Tabela 1, sendo: $\tau_{1}=\tau_{2}=1$, $\gamma_{o}=-\omega_{2}, \varphi_{o}=8\left|\omega_{1}\right|+3 y^{2}+2$ e $\bar{\varphi}_{o}=2\left|\omega_{1}\right|+\left|\omega_{2}\right|+|y|$. Note que, como $v_{1}=y$ é medido, apenas um limitante para a norma de $v_{2}=\dot{y}$ é necessário.

Na Tabela 1, o par $\left(A_{\rho}, b_{\rho}\right)$ está na forma canônica de Brunovsky (com $\rho=2)$ e a trajetória desejada $y_{m}$ é gerada com $k_{m}=4, A_{m}=A_{\rho}+b_{\rho} K_{m}, K_{m}=\left[\begin{array}{ll}-4 & -2\end{array}\right]$ e $r=\operatorname{sgn}(\operatorname{sen}(0.5 \pi t))$. As funções de modulação e dominação são dadas por $\varrho=15\left|\omega_{1}\right|+7.4\left|\omega_{2}\right|+4.4|y|+$ $3 y^{2}+4|r|+2.1$ e $\psi_{\mu}=56\left|\omega_{1}\right|+28\left|\omega_{2}\right|+13|y|+15 y^{2}+22$, respectivamente. Além disso, o HGO e a superfície de deslizamento são implementados com $l_{1}=2, l_{2}=1 \mathrm{e}$ $S=\left[\begin{array}{ll}2 & 1\end{array}\right]$.

Para $y(0)=0$ e $\dot{y}(0)=0$, com um constante e grande valor de $\mu(t)=\bar{\mu}=1$, uma aparente degradação na precisão do rastreamento em malha fechada ( $y$ nem mesmo converge para $\left.y_{m}\right)$ é observado na Fig. 2 (a). Além disso, para $y(0)=5$ e $\dot{y}(0)=0$, a saída da planta escapa em $t \approx 1.79 \mathrm{~s}$ (curva não mostrada). Por outro lado, quando o parâmetro $\mu(t)$ variante no tempo é implementado com 

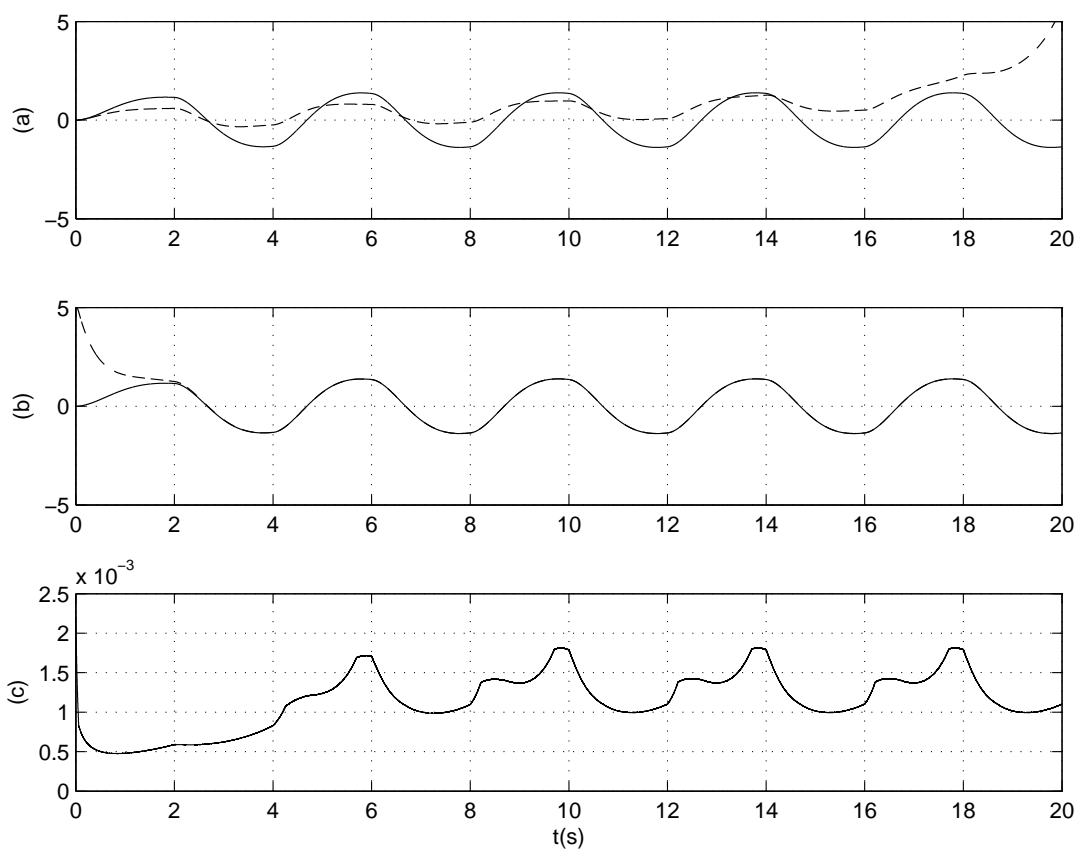

Figura 2: Resultados de Simulação. (a): (linha tracejada) $y$, (linha contínua) $y_{m}$ quando $\mu$ é mantido constante igual a $\mu=1$, com $y(0)=0$ e $\dot{y}(0)=0$; (b): (linha tracejada) $y$, (linha contínua) $y_{m}$ quando $\mu(t)$ é variante no tempo de acordo com (25) com $\bar{\mu}=1, y(0)=5$ e $\dot{y}(0)=0 ;(\mathrm{c})$ : o parâmetro variante no tempo $\mu(t)$.

o mesmo grande valor em $\bar{\mu}=1$, a saída da planta converge para a trajetória desejada a partir de $y(0)=5$, como mostrado na Fig. 2 (b). Neste caso, a evolução de $\mu(t)$ ao longo do tempo é mostrada na Fig. 2 (c), a partir da qual pode-se verificar que uma constante $\mu=\bar{\mu}=0.0005$ poderia ser usada. Entretanto, esse valor não é conhecido a priori. Além do mais, muito cuidado deve ser tomado ao reduzir $\bar{\mu}$, visto que existe um compromisso entre robustez a ruído de medição e a precisão do rastreamento. Até o presente momento, não existe estratégia de controle por modos deslizantes que seja imune a ruído de medição. Isto ainda é mais crítico em esquemas via realimentação de saída baseados em diferenciadores e observadores de alto ganho, como é caso do esquema proposto neste trabalho. Por outro lado, os testes experimentais realizados com esquemas também baseados em alto ganho (Cunha et al., 2005) indicam que a robustez do algoritmo proposto com respeito à ruído de medição é, até certo ponto, aceitável. Apesar disso, uma melhor avaliação da influência do ruído de medição no desempenho do sistema de controle se faz necessária.

Na Fig. 3, o transitório do estado estimado $\hat{\xi}$ é apresentada. A Fig. 3(a) mostra a convergência rápida de $\hat{\xi}_{1}$ para a saída da planta $y$. A Fig. 3(b) mostra o peaking presente em $\hat{\xi}_{2}$ que também converge para $\dot{y}$ após um rápido transitório.

\section{CONCLUSÕES}

O desafiador problema de rastreamento global para sistemas SISO não-lineares incertos e variantes no tempo foi resolvido através do controle por modos deslizantes utilizando somente realimentação de saída. Foi considerada uma ampla classe de plantas que incluem não-linearidades majoradas de forma afim no estado não-medido com taxa de crescimento dependente nãolinearmente de estados internos e da saída medida. Este artigo mostra que é possível aplicar técnicas de dominação e projetar um HGO com ganho dinâmico a fim de obter rastreamento global e prático via controle por modos deslizantes e realimentação de saída, livre de peaking. Acreditamos que esse seja o primeiro resultado global no contexto de SMC para a classe de sistemas considerada.

\section{A CONDIÇÕES GEOMÉTRICAS PARA A FORMA NORMAL}

A fim de considerar explicitamente a dependência do tempo de $f(x, t)$ em (1), seja: $\beta_{k}:=L_{f} \beta_{k-1}+\frac{\partial \beta_{k-1}}{\partial t}+$ $\frac{\partial\left[L_{f}^{k-1} h\right]}{\partial t}$, para $k \in\{1, \ldots, \rho\}$ e $\beta_{0}:=0$. Uma condição suficiente para garantir que a planta variante no tempo 

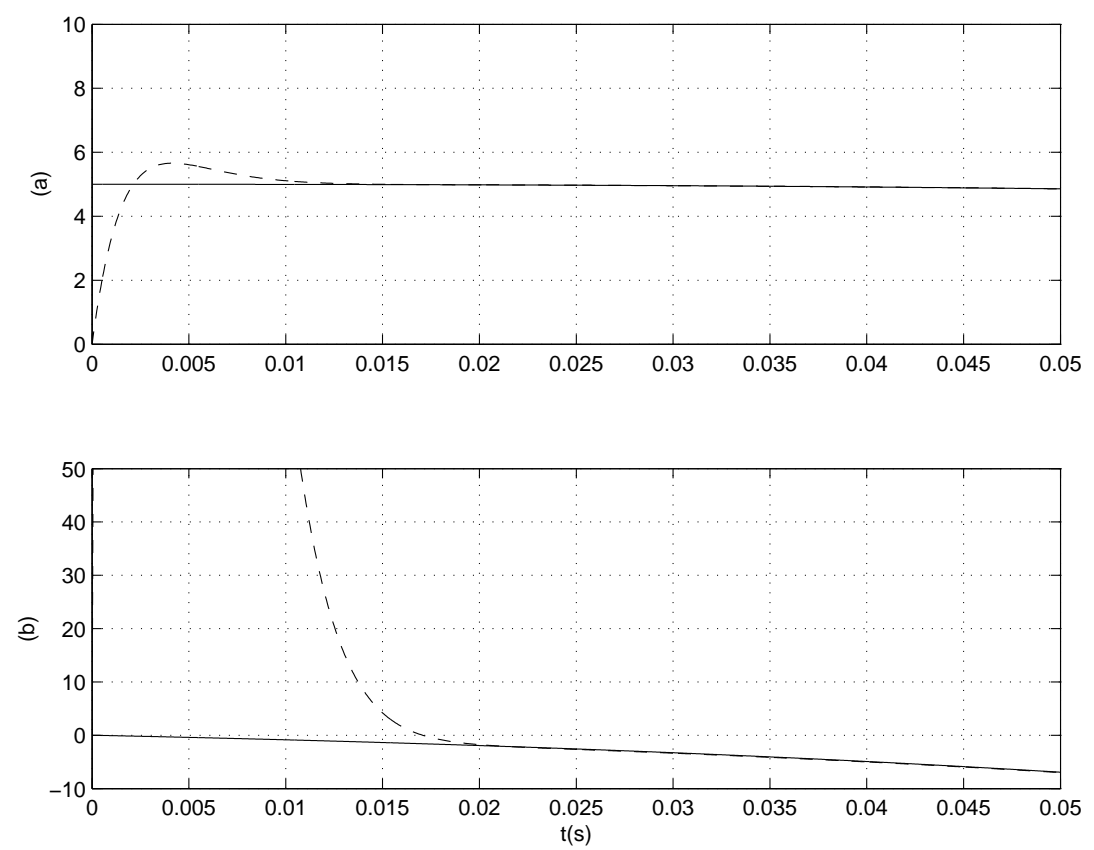

Figura 3: Resultados de Simulação. Estimativas do transitório do HGO (a): (linha contínua) y, (linha tracejada) $\hat{\xi}_{1}$, (b): (linha contínua) $\dot{y}$, (linha tracejada) $\hat{\xi}_{2}$.

(1)-(2) é transformável para a forma normal é dada por: $L_{g}\left[L_{f}^{k} h+\beta_{k}\right] \equiv 0(k \in\{0, \ldots, \rho-2\})$, onde a derivada de Lie de uma função $h$ ao longo de um campo vetorial $f$ é denotada por $L_{f} h$, como em (Khalil, 2002, pp. 510). Neste caso, a transformação $T(x, t)=\left[\eta^{T} T_{\xi}^{T}(x, t)\right]$ é tal que $T_{\xi}:=\left[L_{f}^{0} h+\beta_{0}, L_{f} h+\beta_{1}, \ldots L_{f}^{\rho-1} h+\beta_{\rho-1}\right]^{T}$. Adicionalmente, o HFG da planta $k_{p}(x, t)=L_{g}\left[L_{f}^{\rho-1} h+\right.$ $\left.\beta_{\rho-1}\right]$, a perturbação de entrada $d(x, t)=\left(L_{f}^{\rho} h+\beta_{\rho}\right) / k_{p}$ e $T$ devem satisfazer a Hipótese 1.

\section{B OBSERVADOR DA NORMA}

Neste apêndice, considera-se sistemas na forma (50)-(51) satisfazendo $(\mathrm{C} 0)$ e (C1) da Seção 8. A seguir, nós fornecemos os passos para a obtenção do observador da norma (53)-(55), de acordo com a Definição 1.

\section{- Limitante para $|\eta|$ : obtendo $c_{0}$ e $\varphi_{1}$ em (54)}

A partir de (C1), a função $\alpha_{1}$ é stiffening. Isso garante que $\alpha_{1}(\sigma)>\lambda \sigma, \forall \sigma>\epsilon$, para qualquer $\epsilon>0$ e $0<\lambda<\alpha_{1}(\epsilon) / \epsilon$. Além disso, de (52), pode-se escrever $\dot{V} \leq-\alpha_{1}(V)+\varphi_{\eta}(y, t)$ ou, equivalentemente, $\dot{V} \leq-\lambda V+\left[\lambda V-\alpha_{1}(V)\right]+\varphi_{\eta}(y, t)$. Agora, dado qualquer $V$, ou $V \leq \epsilon$ ou $V>\epsilon$. Consequentemente, ou $\dot{V} \leq-\lambda V+\left[\lambda \epsilon+\alpha_{1}(\epsilon)\right]+\varphi_{\eta}$ ou $\dot{V} \leq-\lambda V+\varphi_{\eta}$, o que nos faz concluir que $\dot{V} \leq-\lambda V+\left[\lambda \epsilon+\alpha_{1}(\epsilon)\right]+\varphi_{\eta}$. Por- tanto, usando o Teorema da Comparação (Khalil, 2002), obtém-se

$$
V \leq e^{-c_{0} t} * \varphi_{1}(y, t)+V(\eta(0)) e^{-c_{0} t},
$$

onde $\varphi_{1}=\varphi_{\eta}+c_{0} \epsilon+\alpha_{1}(\epsilon), c_{0}=\lambda$ são conhecidos $\mathrm{e}$ o operador $*$ denota a convolução pura. Relembrando que $\underline{\lambda}|\eta|^{2} \leq V$, então pode-se obter $|\eta| \leq \sqrt{\left|\omega_{21}\right| / \underline{\lambda}}+\pi_{0}$, com $\omega_{21}$ em (54) e $\pi_{0}$ sendo um termo exponencialmente decrescente dependente de $|\eta(0)|$ e $\left|\omega_{21}(0)\right|$.

- Limitante para $|v|$ : obtendo $\varphi_{2}$ e $\varphi_{3}$ em (55)

Será útil reescrever (51) na forma compacta

$$
\dot{v}=A_{\rho} v+b_{\rho} k_{u} u+\phi(x, t),
$$

onde $\left(A_{\rho}, b_{\rho}\right)$ é o par canônico de Brunovsky e aplicar a mudança de variável $\bar{v}=v-b_{\rho} k_{u} \tau_{1} \omega_{1}$ para obtermos:

$$
\dot{\bar{v}}=A_{\rho} \bar{v}+b_{\rho} k_{u} \omega_{1}+\phi .
$$

Pela observabilidade do $\operatorname{par}\left(A_{\rho}, c_{\rho}\right)$, onde $c_{\rho}=$ $\left[\begin{array}{llll}1 & 0 & \ldots & 0\end{array}\right]$, existe uma matriz $P=P^{T}>0$ e um vetor coluna arbitrário $L$ satisfazendo $A_{L}^{T} P+P A_{L}=-I$, sendo $A_{L}=A_{\rho}-L c_{\rho}$ uma matriz Hurwitz.

Agora, considerando $T_{1}:=\operatorname{diag}\left(1, \varepsilon, \varepsilon^{2}, \ldots, \varepsilon^{\rho-1}\right)$ e uma constante $\varepsilon>0$ qualquer, as seguintes propriedades podem ser checadas: (i) $T_{1} A_{\rho} T_{1}^{-1}=\varepsilon^{-1} A_{\rho}$, (ii) $c_{\rho} T_{1}^{-1}=$ 
$c_{\rho}$ e (iii) $T_{1} b_{\rho}=b_{\rho} \varepsilon^{\rho-1}$. Assim, somando e subtraindo o termo $\left(\varepsilon T_{1}\right)^{-1} L c_{\rho} \bar{v}$ na dinâmica de $\bar{v}$, pode-se escrever $\dot{\bar{v}}=\left[A_{\rho}-\left(\varepsilon T_{1}\right)^{-1} L c_{\rho}\right] \bar{v}+b_{\rho} k_{u} \omega_{1}+\phi+\left(\varepsilon T_{1}\right)^{-1} L y$. Além disso, aplicando-se a transformação $\vartheta=T_{1} \bar{v}$ e considerando as propriedades (i)-(iii) acima, tem-se que

$$
\dot{\vartheta}=\varepsilon^{-1} A_{L} \vartheta+b_{\rho} \varepsilon^{\rho-1} k_{u} \omega_{1}+\varepsilon^{-1} L y+T_{1} \phi .
$$

O passo chave é notar que, devido à condição de triangularidade $(\mathrm{C} 0)$ :

$$
\left|T_{1} \phi\right| \leq k_{\vartheta} \varphi_{r}|\vartheta|+\varphi_{\vartheta},
$$

onde $k_{\vartheta}$ é independente de $\varepsilon$. Em seguida, utilizando-se a derivada de $\operatorname{Dini}^{4}$ e o limitante superior $\Psi_{v}$ dado em (C0), a derivada temporal de $V:=\left(\vartheta^{T} P \vartheta\right)^{1 / 2}$ ao longo da solução da dinâmica de $\vartheta$ satisfaz

$$
\dot{V} \leq-\frac{c_{1}}{\varepsilon} V+c_{2} \varphi_{r} V+\bar{\varphi}_{1}\left(\omega_{21}, \omega_{1}, y, t, \varepsilon\right)+\pi_{1},
$$

onde $\pi_{1}$ é um termo exponencialmente decrescente e a função não-negativa $\bar{\varphi}_{1}$, assim como, as constantes nãonegativas $c_{1}, c_{2}$ são todas conhecidas, satisfazendo $c_{1} \leq$ $1 /\left(2 \lambda_{\max }(P)\right), c_{2} \geq|P| k_{\vartheta} / \lambda_{\min }(P)$ e $\left[\mid b_{\rho} \varepsilon^{\rho-1} k_{u} \omega_{1}+\right.$ $\left.\varepsilon^{-1} L y \mid+\varphi_{\vartheta}\right] c_{3} \leq \bar{\varphi}_{1}+\pi_{1}, \operatorname{com} c_{3} \geq|P| / \sqrt{\lambda_{\min }(P)}$.

Portanto, dado qualquer $V$, ou

$$
V \leq \bar{\varphi}_{1} \quad \text { ou } \quad \dot{V} \leq-\frac{c_{1}}{\varepsilon} V+c_{2} \varphi_{r} V+V+\pi_{1} .
$$

Agora, considere

$$
\bar{\varphi}_{4}\left(\omega_{21}, y, t\right):=\varphi_{2}\left(\omega_{21}\right)+\varphi_{3}(y, t),
$$

com as funções não-negativas $\varphi_{2}, \varphi_{3}$ em (55) a serem determinadas. Assim, (55) pode ser escrita como

$$
\dot{\omega}_{22}=-\frac{1}{\tau_{2}} \gamma\left(\omega_{22}\right)+\bar{\varphi}_{4},
$$

como $\gamma(\sigma):=1-e^{-\sigma}$. Consequentemente, utilizando-se a função limitante $\Psi_{r}$, dada em $(\mathrm{C} 0)$, devemos escolher $\bar{\varphi}_{4} \mathrm{em}(61)$ (e as funções $\varphi_{2}, \varphi_{3}$ ) a fim de satisfazer:

$$
c_{2} \varphi_{r}+1 \leq \bar{\varphi}_{4}\left(\omega_{21}, y, t\right) .
$$

\section{- Limitante para $|v|$}

O limitante para o subsistema- $v$ pode ser obtido considerando-se dois casos para a taxa de crescimento $\varphi_{r}(|\eta|, y, t): \varphi_{r}>k_{r}$ e $\varphi_{r} \leq k_{r}$, onde $k_{r}=3 /\left(c_{2} \tau_{2}\right)$ e $\tau_{2}$ é a constante positiva de projeto em (62).

\footnotetext{
${ }^{4}$ Para evitarmos a derivada de Dini, poderíamos ter utilizado a relação $a b \leq a^{2}+b^{2}$, válida $\forall a, b>0$, à custa de algum conservadorismo.
}

Caso 1: Neste caso, tem-se $3 / \tau_{2} \leq c_{2} k_{r}+1 \leq c_{2} \varphi_{r}+$ $1 \leq \bar{\varphi}_{4}$. Deste modo, pode-se verificar que

$$
\gamma(\sigma) \leq 2 \leq \tau_{2} \bar{\varphi}_{4}-1, \quad \forall \sigma .
$$

Agora, considere $W:=\ln (V+1)$ (Kaliora et al., 2006). Então, $\dot{W}=\dot{V} /(V+1)$ e, a partir de $(60)$, pode-se escrever

$$
V \leq \bar{\varphi}_{3} \quad \text { or } \quad \dot{W} \leq-\frac{1}{\tau_{2}} \gamma(W)+\bar{\varphi}_{4}+\pi_{1},
$$

$\operatorname{com} \varepsilon=c_{1} \tau_{2}, \bar{\varphi}_{3}:=\left.\bar{\varphi}_{1}\right|_{\varepsilon=c_{1} \tau_{2}}$ e notando-se que $V /(V+$ 1), $1 /(V+1) \leq 1$.

Agora, dado qualquer $W$, temos duas possibilidades: $W<\omega_{22}$ ou $W \geq \omega_{22}$. Considerando o último caso, podese escrever $-\gamma\left(\omega_{22}\right) \geq-\gamma(W)$, visto que $\gamma$ é uma função crescente. Por essa razão, a partir de (64) e (62), tem-se que $\dot{\omega}_{22} \geq \dot{W}-\pi_{1}$. Além do mais, de (63), $\dot{\omega}_{22}$ também satisfaz $\dot{\omega}_{22} \geq 1 / \tau_{2}$. Consequentemente, somando as duas últimas desigualdades obtém-se

$$
\dot{W}-2 \dot{\omega}_{22} \leq-\frac{1}{\tau_{2}}+\pi_{1}
$$

Recordando que $\pi_{1}=\beta_{1} e^{-\lambda_{1} t}$ e fazendo $\bar{W}=W+$ $\pi_{1} / \lambda_{1}$, para alguma constante positiva $\lambda_{1}$ e $\beta_{1} \in \mathcal{K}_{\infty}$. Então, tem-se $\dot{\bar{W}}-2 \dot{\omega}_{22} \leq-\frac{1}{\tau_{2}}$, a partir do qual pode-se concluir que, $\bar{W} \leq 2 \omega_{22}-t / \tau_{2}+\left|\bar{W}(0)-2 \omega_{22}(0)\right|$. Note que, é sempre possível encontrar um termo exponencialmente decrescente que seja um majorante para a função afim no tempo acima, i.e., $-t / \tau_{2}+\left|\bar{W}(0)-2 \omega_{22}(0)\right| \leq \pi_{2}$, onde $\pi_{2}:=\beta_{2}\left(|\bar{W}(0)|+\left|\omega_{22}(0)\right|\right) e^{-\lambda_{2} t}, \operatorname{com} \beta_{2} \in \mathcal{K}_{\infty} \mathrm{e}$ alguma constante $\lambda_{2}>0$. Finalmente, dado $W$, pode-se concluir que $W \leq 2\left|\omega_{22}\right|+\pi_{2}+\pi_{1} / \lambda_{1}$ e, utilizando o Teorema da Comparação (Khalil, 2002) e relembrando que $V=e^{W}-1$, podemos escrever

$$
V \leq e^{2\left|\omega_{22}\right|}+\pi_{3},
$$

sendo $\pi_{3}$ um termo exponencial decrescente.

Caso 2: Assuma que $\varphi_{r} \leq k_{r}$ e faça $\varepsilon=c_{1} /\left(c_{2} k_{r}+2\right)$ em (60). Desta forma, pode-se escrever:

$$
V \leq \bar{\varphi}_{2} \quad \text { or } \quad \dot{V} \leq-V+\pi_{1},
$$

na qual $\bar{\varphi}_{2}=\left.\bar{\varphi}_{1}\right|_{\varepsilon=c_{1} /\left(c_{2} k_{r}+2\right)}$. Neste caso, somando os dois limitantes obtidos de (66), podemos escrever também

$$
V \leq \bar{\varphi}_{2}+\pi_{4},
$$

sendo $\pi_{4}$ um termo exponencial decrescente. Assim, a partir de (65) e (67) tem-se

$$
V \leq e^{2\left|\omega_{22}\right|}+\bar{\varphi}_{1}\left(\omega_{21}, \omega_{1}, y, t, \varepsilon\right)+\pi_{5},
$$


com $\varepsilon=c_{1} /\left(3 / \tau_{2}+2\right)$ e utilizando-se a desigualdade de Rayleigh, pode-se obter um limitante superior para $v$.

Finalmente, juntando-se os majorantes para $|v|$ e $|\eta|$, podemos obter a função não-negativa $\varphi_{4} \mathrm{e}$ as constantes não-negativas em (56).

\section{PROVAS AUXILIARES}

- Demonstrações das Desigualdades (15), (16) e (17)

Do observador da norma do estado da planta, tem-se $|x| \leq \bar{\varphi}_{o}(\omega, t)+\pi_{o}$. Note que, para qualquer função crescente $\psi: \mathbb{R}^{+} \rightarrow \mathbb{R}^{+}$, pode-se escrever $\psi(a+b) \leq$ $\psi(2 a)+\psi(2 b), \forall a, b \in \mathbb{R}^{+}$. Visto que $\varphi_{i}(i=1,2,3)$ são funções não-negativas e crescentes em seu primeiro argumento, então $\varphi_{i}(|x|, y, t) \leq \varphi_{i}\left(2 \bar{\varphi}_{o}, y, t\right)+\varphi_{i}\left(2 \pi_{o}, y, t\right)$. Além disso, pode-se também concluir da Hipótese 1 que $\varphi_{i}\left(2 \pi_{o}, y, t\right) \leq \alpha_{i}\left(2 \pi_{o}\right)+\bar{\varphi}_{i}(y, t)$ e, visto que $\alpha_{i} \in \mathcal{K}$ são funções localmente Lipschitz, $\alpha_{i}\left(2 \pi_{o}\right) \leq \pi_{1}$, onde $\pi_{1}=\beta_{1}(|\omega(0)|+|x(0)|) e^{-\lambda_{o} t}$ com algum $\beta_{1} \in \mathcal{K}_{\infty}$. Portanto, pode-se escrever $\varphi_{i}(|x|, y, t) \leq \psi_{i}(\omega, t)+\pi_{1}$, com $\psi_{i}(\omega, t):=\varphi_{i}\left(2 \bar{\varphi}_{o}, y, t\right)+\bar{\varphi}_{i}(y, t)$. Recordando que $\left[\begin{array}{ll}\eta^{T} & \xi^{T}\end{array}\right]^{T}=T(x, t)$, então $|\xi| \leq|T(x, t)|$. Assim, da Hipótese 1, tem-se $|\xi| \leq \varphi_{1}(|x|, y, t)$. Consequentemente, $\xi, k_{p}$ e $d$ satisfazem (15), (16) e (17).

- Demonstrações das Desigualdades (44) e (45)

Se $\beta_{4}(|\omega(0)|+|x(0)|) \leq 1$ ou $t_{M}$ é infinito, a demonstração é trivial devido ao termo exponencial decrescente $e^{-\lambda_{4} t}$. Agora, considere que $\beta_{4}(|\omega(0)|+|x(0)|)>1 \mathrm{e}$ $t_{M}$ seja finito. Então, tem-se: (i) $e^{-\lambda_{\mu} t} \geq e^{-\lambda_{\mu} t_{M}}, \forall t \in$ $\left[0, t_{M}\right)$; (ii) $\exists t_{1} \in\left[0, t_{M}\right)$ tal que $\left\|\omega_{t}\right\| \geq \delta, \forall t \in\left[t_{1}, t_{M}\right)$, onde $\delta$ é uma constante arbitrária. Portanto, de (i) e (ii) e considerando $\delta \geq\left(\beta_{4}-1\right) e^{\lambda_{\mu} t_{M}}$, tem-se também que o lado direito de (43) é limitado por $\bar{\mu}$. Além disso, durante o intervalo $\left[0, t_{\mu}\right)$, por definição de $t_{\mu}$, tem-se que $|\omega(t)| \leq \beta_{5}(|\omega(0)|+|x(0)|)$. Notando que (i) $e^{\lambda_{\mu} t_{\mu}}$ pode ser majorado por uma função classe- $\mathcal{K}$ de $|\omega(0)|+|x(0)|$ e (ii) $\zeta$ (31) escapa no máximo exponencialmente, podemos concluir que $|\zeta|$ e $|\omega|$ podem ser majorados por uma função classe- $\mathcal{K}$ de $|\omega(0)|+|x(0)|+|\zeta(0)|$.

\section{- Provando que (47) Satisfaz (P1)}

Primeiramente, note que para qualquer função absolutamente contínua $g(t),\left\|g_{t}\right\|=|g(t)|$ ou $\left\|g_{t}\right\|$ é uma constante positiva. Portanto, $\left|\frac{d\left\|g_{t}\right\|}{d|g|}\right| \leq 1$, quase sempre (almost everywhere), consequentemente, $\left|\frac{\partial \psi_{\mu}}{\partial|\omega|}\right| \leq$ $\left|\frac{d p_{\mu}(|\omega|)}{d|\omega|}\right|+e^{-\lambda_{\mu} t}$. Além disso, uma vez que $d p(a) / d a \leq$ $k_{1} p(a)$, onde $p(a)$ é qualquer polinômio em $a$ com coeficientes reais positivos e $k_{1}$ é uma constante apropriada, pode-se também escrever que $\left|\frac{\partial \psi_{\mu}}{\partial|\omega|}\right| \leq k_{1} p_{\mu}(|\omega|)+e^{-\lambda_{\mu} t}$. Como $\left|\frac{\partial|\omega|}{\partial \omega}\right| \leq 1$, então $\left|\frac{\partial \psi_{\mu}}{\partial \omega}\right| \leq\left|\frac{\partial \psi_{\mu}}{\partial|\omega|}\right|$ e pode-se concluir que (P1) é satisfeita, visto que $\frac{\partial \psi_{\mu}}{\partial t}=-\lambda_{\mu}\left\|\omega_{t}\right\| e^{-\lambda_{\mu} t}$.

\section{PROVAS PRINCIPAIS}

\section{- Prova do Lema 1}

Introduzindo a transformação de coordenadas $\bar{\xi}_{e}=T_{n} \xi_{e}$, $\operatorname{com} T_{n}:=\left[\begin{array}{ll}I & S^{T}\end{array}\right]^{T}$, o sistema (13) pode ser levado para a forma normal. Logo, pode-se concluir que (13) é OSS com respeito à saída de grau relativo unitário $S \xi_{e}$, ou seja, $\xi_{e}$ e $e$ satisfazem

$$
\left|\xi_{e}\right|,|e| \leq k_{1}\left|S \xi_{e}\right|+\pi_{1},
$$

com $k_{1}>0$ sendo uma constante e $\pi_{1}=\beta_{1}\left(\left|\xi_{e}(0)\right|\right) e^{-\lambda_{m} t}$ um termo exponencialmente decrescente com alguma função $\beta_{1} \in \mathcal{K}_{\infty}$ e $0<\lambda_{m}<\lambda_{m}\left[A_{m}\right]$. Note que, para todo $\tilde{\xi}_{e}$, ou $\left|S \xi_{e}\right| \leq\left|S \tilde{\xi}_{e}\right|$ ou $\left|S \xi_{e}\right|>\left|S \tilde{\xi}_{e}\right|$. Sendo as$\operatorname{sim}$, ou $\left|S \xi_{e}\right| \leq\left|S \tilde{\xi}_{e}\right|$ ou $\operatorname{sgn}(\hat{\sigma})=\operatorname{sgn}\left(S \xi_{e}\right)$. Considere o último caso.

Utilizando-se a função de energia $V=\xi_{e}^{T} P \xi_{e}$, onde $P=P^{T}>0$ é a solução de $A_{m}^{T} P+P A_{m}=-I$, pode-se assegurar que a derivada temporal de $V$ ao longo das soluções de (13) satisfaz

$$
\dot{V} \leq-\left|\xi_{e}\right|^{2}-2 k_{p}\left|S \xi_{e}\right|\left[\varrho-\left|d_{e}\right|\right] .
$$

Desta forma, como $\varrho$ em (19) satisfaz (18), tem-se que $\dot{V} \leq-\left|\xi_{e}\right|^{2}$, que juntamente com o caso $\left|S \xi_{e}\right| \leq\left|S \tilde{\xi}_{e}\right|$ leva à desigualdade $\left|S \xi_{e}\right| \leq\left|S \tilde{\xi}_{e}\right|+\pi_{2}$, onde $\pi_{2}$ é um termo exponencialmente decrescente. Conseqüentemente, a partir de (69), a dinâmica que governa $\xi_{e}$ é ISS com respeito a $\tilde{\xi}_{e}$.

\section{- Prova do Teorema 1}

Passo-1: A partir da Definição 1, da Hipótese 1 e de (44), pode-se verificar que $|z(t)| \leq \beta_{1}(|z(0)|)+k_{1}, \forall t \in$ $\left[0, t_{\mu}\right]$, onde $\beta_{1} \in \mathcal{K}_{\infty}$ e $k_{1} \geq 0$ é uma constante.

Passo-2: Considere a dinâmica- $\zeta$ (31) e a função de armazenamento $V=\zeta^{T} P \zeta$, onde $P=P^{T}>0$ é a solução de $A_{o}^{T} P+P A_{o}=-I$. Então, a derivada temporal de $V$ ao longo das trajetórias de (31) satisfaz $\mu \dot{V}=-|\zeta|^{2}+(\dot{\mu})\left[2 \zeta^{T} P \Delta \zeta\right]+(\mu \nu)\left[2 \zeta^{T} P b_{\rho}\right]$. Agora, projetando $\mu$ para satisfazer (P0)-(P2), (45) é válida e a 
seguinte desigualdade é verificada $\forall t \in\left[t_{\mu}, t_{M}\right): \mu \dot{V} \leq$ $-|\zeta|^{2}+\mathcal{O}(\bar{\mu}) k_{1}|\zeta|^{2}+\mathcal{O}(\bar{\mu}) k_{2}|\zeta|$, sendo $k_{1}:=2|P||\Delta|$ e $k_{2}:=2|P|\left|b_{\rho}\right|$. Além disso, visto que $a b<a^{2}+b^{2}$, para quaisquer números $a, b$ reais positivos, então

$$
\mu \dot{V} \leq-\left[1-\mathcal{O}(\bar{\mu}) k_{1}-\mathcal{O}(\bar{\mu})\right]|\zeta|^{2}+\mathcal{O}(\bar{\mu}),
$$

da qual pode-se concluir que $\mu \dot{V} \leq-\lambda_{1} V+\mathcal{O}(\bar{\mu})$, com uma constante apropriada $\lambda_{1}>0$. Agora, seja $V \leq$ $2 \mathcal{O}(\bar{\mu}) / \lambda_{1}$ ou $\mu \dot{V} \leq-\lambda_{1} V / 2$. Considere o último caso. Como $\mu<\bar{\mu}, \log$ o $\dot{V} \leq-\lambda_{1} V /(2 \bar{\mu})$. Consequentemente, conclui-se que $|\zeta|,\left|\tilde{\xi}_{e}\right| \leq \beta_{2}(|\zeta(0)|) e^{-\lambda_{2} t}+\mathcal{O}(\bar{\mu}), \forall t \in$ $\left[t_{\mu}, t_{M}\right)$, com uma constante apropriada $\lambda_{2}>0$ e algum $\beta_{2} \in \mathcal{K}_{\infty}$. Na última desigualdade, o limitante para $\left|\tilde{\xi}_{e}\right|$ foi obtido notando-se que $\tilde{\xi}_{e}=T_{\mu}^{-1} \zeta$ implica em $\left|\tilde{\xi}_{e}\right| \leq|\zeta|$, visto que $\left|T_{\mu}^{-1}\right| \leq 1$ para $\mu<1$.

Passo-3: A partir do Lema 1, existe uma propriedade ISS de $\left|\tilde{\xi}_{e}\right|$ para $\xi_{e}$ e, considerando o majorante dado no Passo-1, pode-se concluir que $\left|\xi_{e}\right|,|z(t)| \leq$ $\left[\beta_{3}(|z(0)|)+k_{3}\right] e^{-\lambda_{3} t}+\mathcal{O}(\bar{\mu}), \forall t \in\left[0, t_{M}\right)$, com constantes apropriadas $\lambda_{3}>0, k_{3} \geq 0$ e algum $\beta_{3} \in \mathcal{K}_{\infty}$. Assim, $|z(t)|$ não pode escapar em tempo finito, sendo uniformemente limitado em $\mathcal{I}:=\left[0, t_{M}\right)(\mathrm{UBI})$.

Passo-4: Uma vez que $z(t)$ é UBI , então $\xi_{e}, \sigma=S \xi_{e}, \zeta$ e $\xi=\xi_{e}+\xi_{m}$ são UBI e, a partir da Hipótese $2, \eta, \bar{x}$ são também UBI. Além disso, de acordo com o limitante inferior para $|T(x, t)|$ dado na Hipótese 1 tem-se que $x$ é UBI. Assim, as funções limitantes superiores dadas na Hipótese 1 garantem que $d, k_{p}, d_{e}$ são também UBI. Agora, reescrevendo (13) na forma normal, pode-se escrever $\dot{\sigma}=-\lambda_{4} \sigma+k_{4}\left(u+d_{e}\right)$, para algumas constantes $\lambda_{4}, k_{4}>0$. Além disso, pela linearidade da solução da última equação, pode-se também escrever $\sigma=\sigma_{1}+\sigma_{2}$, onde $\dot{\sigma}_{1}=-\lambda_{4} \sigma_{1}+k_{4} u$ e $\dot{\sigma}_{2}=-\lambda_{4} \sigma_{2}+k_{4} d_{e}$, com condições iniciais apropriadas. Portanto, visto que $\sigma$ e $d_{e}$ são UBI , $\sigma_{2}$ e $\sigma_{1}$ também são. Neste caso, qualquer sinal satisfazendo $\dot{\sigma}_{3}=-\lambda_{5} \sigma_{3}+k_{5} u$, onde $\lambda_{5}, k_{5}>0$ são constantes, é também UBI , em particular, $\omega_{1}$ definido em (3). Como $y, \omega_{1}$ são UBI e $\varphi_{o}$ é contínuo por partes em seus argumentos, então a dinâmica- $\omega_{2}$, na Definição 1 , não pode escapar em tempo finito. Finalmente, podese concluir que todos os sinais do sistema não podem escapar em tempo finito, i.e., $t_{M} \rightarrow \infty$. Assim sendo, a partir do Passo-3, pode-se verificar diretamente que o sistema do erro é GAS com respeito a um conjunto compacto $\{z:|z| \leq b\}$ e que $z(t)$ é exponencialmente convergente para um conjunto residual de ordem $\mathcal{O}(\bar{\mu})$.

Todos os sinais em malha fechada são uniformemente limitados: Podemos subsequentemente concluir que $|\xi|, y$, $|\eta|,|x|, \sigma_{1}$ e $\omega_{1}$ convergem para um conjunto de ordem $\mathcal{O}\left(|r|+k_{5}\right)$ após um tempo finito, onde $k_{5}$ é uma constante positiva dependente das perturbações variantes no tempo. Assim, existe $\tau_{2}$ suficientemente pequeno e independente das condições iniciais, que assegura que $\omega_{2}$ é limitado após algum tempo finito. Finalmente, pode-se concluir que todos os sinais do sistema são UB $\forall t$.

\section{- Prova do Corolário 1}

Relembrando-se que $A_{\rho}=A_{m}-b_{\rho} K_{m}, \hat{\xi}=\hat{\xi}_{e}+\xi_{m}$, $\hat{\xi}=\xi_{e}+\xi_{m}-\tilde{\xi}_{e}, \hat{\xi}_{e}=\xi_{e}-\tilde{\xi}_{e}$ e $\tilde{\xi}_{e}=T_{\mu}^{-1} \zeta$, pode-se verificar a partir de (23) que $\dot{\hat{\xi}}_{e}=A_{m} \hat{\xi}_{e}+b_{\rho} u+\varsigma_{m}+\varsigma_{e}$, onde $\varsigma_{m}=$ $-b_{\rho}\left(K_{m} \xi_{m}+k_{m} r\right)$ e $\varsigma_{e}=\left(b_{\rho} K_{m}+H_{\mu} L_{o} c_{\rho}\right)\left(\tilde{\xi}_{e}-\xi_{e}\right)+H_{\mu} L_{o} e$. Note que, de acordo com o Teorema 1, todos os sinais do sistema em malha fechada são uniformemente limitados em norma e $z(t) \rightarrow \mathcal{O}(\bar{\mu})$. Então, existe um tempo finito $T_{1}>0$ tal que $\left|\varsigma_{e}\right| \leq \delta_{1}, \forall t \geq T_{1}$, e qualquer $\delta_{1}>0$. Agora, considere a função de energia $V=\hat{\xi}_{e}^{T} P \hat{\xi}_{e}$, onde $P=P^{T}>0$ é a solução de $A_{m}^{T} P+$ $P A_{m}=-Q$, com $Q=Q^{T}>0$ e $P b_{\rho}=S^{T}$ (lembrar que o sistema $\left(A_{m}, b_{\rho}, S\right)$ é estritamente real positivo). Então, calculando $\dot{V}$ ao longo das soluções da dinâmica que governa $\hat{\xi}_{e}$, pode-se verificar que a condição para a existência de modo deslizante $\hat{\sigma} \dot{\hat{\sigma}}<0$ é verificada para algum tempo finito $T_{2} \geq T_{1}$ desde que $\varrho \geq \varsigma_{m}+\delta$, onde $\delta>0$ é uma constante arbitrária.

\section{AGRADECIMENTOS}

Este trabalho contou com o apoio financeiro da FAPERJ, da CAPES e do CNPq.

\section{REFERÊNCIAS}

Ahrens, J. H. \& Khalil, H. K. (2007). Closed-loop behavior of a class of nonlinear systems under ekf-based control, IEEE Trans. Aut. Contr. 52(3): 536-540.

Andrieu, V., Praly, L. \& Astolfi, A. (2007). Asymptotic tracking of a state trajectory by output-feedback for a class of non linear systems, $C D C$, New Orleans (LA), pp. 5228-5233.

Andrieu, V., Praly, L. \& Astolfi, A. (2009). High gain observers with updated gain and homogeneous correction terms, Automatica 45(2): 422-428.

Angeli, D. \& Sontag, E. (1999). Forward completeness, unboundedness observability, and their Lyapunov characterizations, Systems \& Contr. Letters 38(5): 209-217.

Arcak, M., Larsen, M. \& Kokotović, P. (2002). Boundedness without absolute stability in systems with stiffening nonlinearities, European J. of Control. 8(3): 243-250.

Bondarev, A. G., Bondarev, S. A., Kostyleva, N. E. \& Utkin, V. I. (1985). Sliding modes in systems with asymptotic state observers, Autom. Remote Control 46(6): 679-684.

Choi, H.-L. \& Lim, J.-T. (2005). Global exponential stabilization of a class of nonlinear systems by output feedback, IEEE Trans. Aut. Contr. 50(2): 255-257. 
Cunha, J. P. V. S., Costa, R. R., Lizarralde, F. \& Hsu, L. (2009). Peaking free variable structure control of uncertain linear systems based on a high-gain observer, Automatica 45: 11561164 .

Cunha, J. P. V. S., Hsu, L., Costa, R. R. \& Lizarralde, F. (2005). Controle de sistemas lineares incertos por modos deslizantes e observador de alto ganho sem peaking, Controle \& Automação 16(4): 499-466.

Diao, Y. \& Passino, K. M. (2001). Adaptive control fo a class of nonlinear time-varying systems, Proc. American Contr. Conf., Arlington, pp. 4161-4166.

Edwards, C., Colet, E. F. \& Fridman, L. (eds) (2006). Advances in Variable Structure and Sliding Mode Control, SpringerVerlag.

Edwards, C. \& Spurgeon, S. K. (1998). Sliding Mode Control: Theory and Applications, Taylor \& Francis Ltd.

Esfandiari, F. \& Khalil, H. K. (1992). Output feedback stabilization of fully linearizable systems, Int. J. Contr. 56: 10071037.

Filippov, A. F. (1964). Differential equations with discontinuous right-hand side, American Math. Soc. Translations 42(2): 199-231.

Hsu, L. (1997). Smooth sliding control of uncertain systems based on a prediction error, Int. Journal of Robust and Nonlinear Control 7: 353-372.

Hsu, L., Cunha, J. P. V. S., Costa, R. R. \& Lizarralde, F. (2002). Multivariable output-feedback sliding mode control, in X. Yu \& J.-X. Xu (eds), Variable Structure Systems: Towards the 21st Century, Springer-Verlag, pp. 283-313.

Hsu, L., Peixoto, A. J., Cunha, J. P. V. S., Costa, R. R. \& Lizarralde, F. (2006). Output feedback sliding mode control for a class of uncertain multivariable systems with unmatched nonlinear disturbances, Advances in Variable Structure and Sliding Mode Control, Springer-Verlag, pp. 195-225.

Isidori, A. (1995). Nonlinear Control Systems, $3^{\text {rd }}$ edn, Springer.

Jiang, Z. P., Mareels, I., Hill, D. J. \& Huang, J. (2004). A unifying framework for global regulation nonlinear output feedback: from ISS to iISS, IEEE Trans. Aut. Contr. 49(4): 549-562.

Kaliora, G., Astolfi, A. \& Praly, L. (2006). Norm estimators and global output feedback stabilization of nonlinear systems with ISS inverse dynamics, IEEE Trans. Aut. Contr. 51(3): 493498.

Khalil, H. K. (2002). Nonlinear Systems, $3^{\text {rd }}$ edn, Prentice Hall.

Krishnamurthy, P., Khorrami, F. \& Chandra, R. S. (2003). Global high-gain-based observer and backstepping controller for generalized output-feedback canonical form, IEEE Trans. Aut. Contr. 48(12): 2277-2284.

Krishnamurthy, P., Khorrami, F. \& Jiang, Z. P. (2002). Global output feedback tracking for nonlinear systems in generalized output-feedback canonical form, IEEE Trans. Aut. Contr. 47(5): 814-819.

Lei, H. \& Lin, W. (2005). Universal output feedback control of nonlinear systems with unknown growth rate, Proceedings of the $16^{\text {th }}$ IFAC World Congress, Prague, Czech Republic.

Levant, A. (2003). Higher-order sliding modes, differentiation and output-feedback control, Int. J. Contr. 76(9): 924-941.

Marino, R. \& Tomei, P. (1995). Nonlinear Control Design. Geometric, Adaptive and Robust, $1^{\text {st }}$ edn, Prentice Hall.

Mazenc, F., Praly, L. \& Dayawansa, W. (1994). Global stabilization by output feedback: examples and counterexamples, Systems \& Contr. Letters 23: 119-125.
Oh, S. \& Khalil, H. K. (1997). Nonlinear output-feedback tracking using high-gain observer and variable structure control, Automatica 33(10): 1845-1856.

Oliveira, T. R., Peixoto, A. J. \& Hsu, L. (2009). Dwell-time and monitoring schemes for peaking avoidance in high-gain observer based output-feedback control, Proc. IEEE Conf. on Decision and Control, Shanghai, pp. 7557-7562.

Oliveira, T. R., Peixoto, A. J. \& Hsu, L. (2010a). Controle por realimentação de saída para sistemas incertos fortemente nãolineares, Controle \& Automação 21: 69-81.

Oliveira, T. R., Peixoto, A. J. \& Hsu, L. (2010b). Sliding mode control of uncertain multivariable nonlinear systems with unknown control direction via switching and monitoring function, IEEE Trans. Aut. Contr. 55(4): 1028-1034.

Oliveira, T. R., Peixoto, A. J., Nunes, E. V. L. \& Hsu, L. (2007). Control of uncertain nonlinear systems with arbitrary relative degree and unknown control direction using sliding modes, Int. J. Adaptive Control Signal Process. 21: 692-707.

Peixoto, A. J. (2007). Rastreamento de Trajetória por Modos Deslizantes de uma Classe de Sistemas Não-lineares Incertos via Realimentação de Saída, PhD thesis, Ph.D. thesis, Programa de Engenharia Elétrica, COPPE/UFRJ, Rio de Janeiro.

Peixoto, A. J., Hsu, L., Costa, R. R. \& Lizarralde, F. (2007). Global tracking sliding mode control for uncertain nonlinear systems based on variable high gain observer, Proc. IEEE Conf. on Decision and Control, New Orleans, pp. 2041-2046.

Praly, L. (2001). Asymptotic stabilization via output feedback for lower triangular systems with output dependent incremental rate, Proc. IEEE Conf. on Decision and Control, Orlando, pp. 3808-3813.

Praly, L. \& Jiang, Z. (2004). Linear output feedback with dynamic high gain for nonlinear systems, Systems \& Contr. Letters 53: $107-116$.

Qian, C. \& Lin, W. (2002). Output feedback control of a class of nonlinear systems: a nonseparation principle paradigm, IEEE Trans. Aut. Contr. 47(10): 1710-1715.

Sabanovic, A., Fridman, L. \& Spurgeon, S. K. (eds) (2004). Variable Structure Systems: From Principles to Implementation (IEE Control Engineering), Academic Press, Inc.

Sontag, E. D. \& Wang, Y. (1995). On characterizations of the input-to-state stability property, Systems \& Contr. Letters 24: 351-359.

Sontag, E. D. \& Wang, Y. (1997). Output-to-state stability and detectability of nonlinear systems, Systems $\&$ Contr. Letters 29: $279-290$.

Sussmann, H. J. \& Kokotović, P. V. (1991). The peaking phenomenon and the global stabilization of nonlinear systems, IEEE Trans. Aut. Contr. 36(4): 424-440.

Utkin, V. I. (1992). Sliding Modes in Control and Optimization, Springer-Verlag.

Yu, X. \& Xu, J.-X. (eds) (2002). Variable Structure Systems: Towards the 21st Century, Springer-Verlag. 